\title{
Historia y ritual dinásticos en Machaquilá (Petén, Guatemala)
}

\author{
Alfonso LaCADENA GARCÍA-GALLO \\ Dep. de Historia de América II (Antropología de América) \\ Universidad Complutense de Madrid \\ alacaden@ghis.ucm.es
}

Recibido: 15 de octubre de 2010

Aceptado: 19 de octubre de 2010

\section{RESUMEN}

El trabajo se centra en la dinastía real de Machaquilá (Petén, Guatemala), enfocándose en dos aspectos principales: por un lado, la historia política de la dinastía, reconstruida a partir de los textos jeroglíficos disponibles; por otro, los rituales realizados en la Plaza A, donde se encuentra el conocido recinto cuadrilobulado, y que constituyen un testimonio excepcional de las actividades ceremoniales vinculada a la élite gobernante.

Palabras clave: Cultura maya, periodo Clásico, Machaquilá, epigrafía maya, ritual dinástico, cuadrilobulado.

\section{Dynastic History and Ritual in Machaquilá (Petén, Guatemala)}

\begin{abstract}
The article deals with the royal dynasty of Machaquilá (Petén, Guatemala), focusing on two main aspects: On one side, the political history of the dynasty, reconstructed from the available hieroglyphic texts; on the other, the rituals that took place at Plaza A, where the well-known quatrifoil was found, which represent an exceptional expression of the ceremonial activities of the ruling elite.
\end{abstract}

Key words: Maya Culture, Classic Period, Machaquila, Maya epigraphy, dynastic ritual, quatrefoil.

Sumario: 1. El corpus glífico de Machaquilá. 2. La historia dinástica de Machaquilá según el registro epigráfico. 3. Rituales dinásticos en la Plaza A de Machaquilá. 4. Referencias bibliográficas. Apéndice I: Relación de textos jeroglíficos de Machaquilá. Apéndice II: Secuencia dinástica de Machaquilá.

\section{El corpus glífico de Machaquilá}

El corpus de textos jeroglíficos de Machaquilá se ha ido reuniendo a lo largo del medio siglo transcurrido entre los primeros hallazgos de inscripciones que se producen tras el descubrimiento del sitio en 1957 por empleados de la Union Oil Company, las exploraciones de Ian Graham en los años sesenta, los trabajos del Atlas Arqueológico de Guatemala dirigido por Juan Pedro Laporte desde finales de la década de los noventa, y el final de los trabajos arqueológicos del equipo hispano-guatemalteco (20012002 y 2003-2005) en el contexto del proyecto «La entidad política de Machaquilá (Guatemala) durante el Clásico Tardío y Terminal».

Corresponde a Ian Graham en Archaeological Explorations in El Peten, Guatemala (1967: 51-99) la primera y más extensa publicación de los monumentos labrados del sitio. Su trabajo incluyó la publicación de fotografías y/o dibujos de las estelas 1-16,

Esta investigación se ha realizado en el marco del Proyecto «La entidad política de Machaquilá (Guatemala) durante el Clásico Tardío y Terminal», financiado por el Ministerio de Cultura (2001-2002) y por el Ministerio de Ciencia y Tecnología y Ministerio de Educación (2003-2005, nº de Referencia: BHA 2002-03729). 


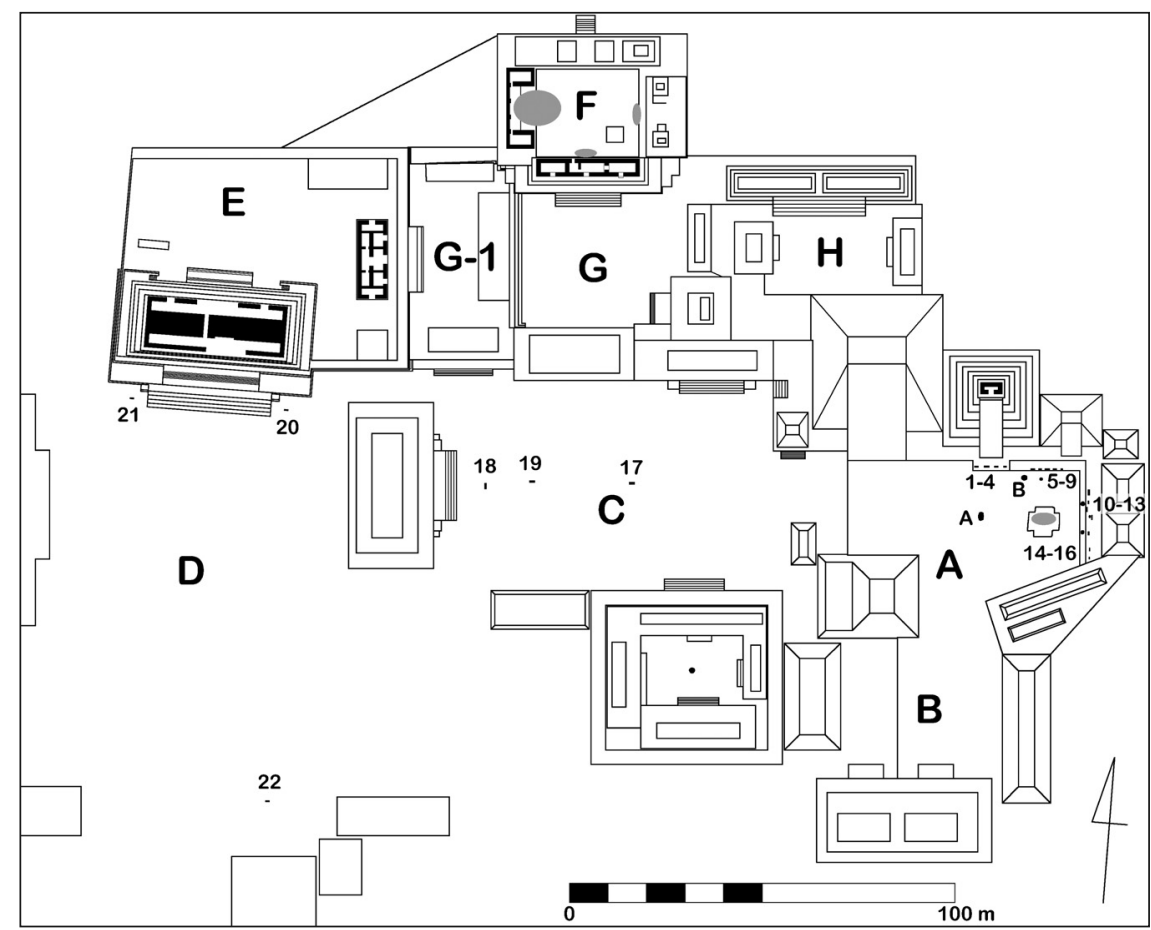

Figura 1: Procedencia de los textos jeroglíficos en Machaquilá (la ubicación de las estelas 18, 19 y 22 es aproximada; los óvalos señalan la zona aproximada de procedencia de los fragmentos glíficos de las estructuras 4, 7-8 y 10 de la Plaza F y del cuadrilobulado de la Plaza A) (según dibujo de J. Adánez).

todas ubicadas en la Plaza A, y la Estela 17, situada en el eje este-oeste de la Plaza C, así como los altares A y B (registrando también los altares lisos C-F) y diversos fragmentos glíficos asociados a la Estructura 4. Con posterioridad, en 1968, Graham documentó fotográficamente las estelas 18 y 19 ubicadas en la Plaza C (vid. Riese 1988a: 98, Nota 2). En los años ochenta del pasado siglo las inscripciones del sitio recibieron la atención por parte del epigrafista guatemalteco Federico Fahsen, quien estableció la primera secuencia dinástica formal del sitio (Fahsen 1983; 1984), incluyendo un dibujo de la Estela 18, por aquel entonces en una colección europea. El epigrafista alemán Berthold Riese $(1984,1988 \mathrm{a}, 1988$ b) se interesó asimismo por la historia dinástica y la cronología del sitio, ofreciendo un nuevo dibujo de la Estela 18 y una revisión de su información calendárica. El también epigrafista alemán Nikolai Grube visitó brevemente el yacimiento a finales de los ochenta, publicando un nuevo fragmento glífico, posiblemente de la Estructura 4 (Grube 1989). Al iniciar sus trabajos en el sitio a finales de los noventa, el Proyecto Atlas Arqueológico de Guatemala realizó un registro del estado y ubicación de los monumentos y fragmentos esculpidos conocidos hasta entonces -confirmando un fuerte saqueo-, y documentando tres nuevas estelas, las tres mutiladas: las estelas 20 y 21, flanqueando la escalinata sur de la Estructura 34 en la Plaza D, y la Estela 22, en el extremo sur de la misma plaza (Chocón y Laporte 2002; Chocón 2003). Finalmente, en el transcurso de los trabajos del Proyecto «La entidad 
política de Machaquilá (Guatemala) durante el Clásico Tardío y Terminal» el corpus glífico del sitio se incrementó con la recuperación de 19 nuevos fragmentos glíficos pertenecientes a la Estructura 4 de la Plaza F (Iglesias y Lacadena 2003; Lacadena e Iglesias 2005; Lacadena 2005) y cinco fragmentos cerámicos procedentes del recinto cuadrilobulado de la Plaza A (Lacadena 2006) (Figura 1 y Apéndice 1).

El grado de conservación de los monumentos e inscripciones de Machaquilá es muy desigual, variando desde algunas de las estelas con el relieve perfectamente conservado, hasta monumentos sumamente deteriorados, como la Estela 19, cuyos textos no son ya legibles. Tras la publicación del estudio de Graham (1967) el sitio arqueológico sufrió un fuerte saqueo. El saqueo afectó también a los monumentos de piedra, algunos de los cuales fueron cortados con sierra mecánica, sustrayéndose las partes con los relieves mejor conservados y desechando los otros, y robando monumentos completos. En la actualidad, el corpus jeroglífico de Machaquilá se encuentra disperso, entre el propio sitio, la Bodega del Atlas Arqueológico de Guatemala en Dolores, Petén, el Museo de Dolores, Petén, el Museo de la Aurora de Ciudad de Guatemala (sala de exposición permanente y bodega) y diversas colecciones privadas.

\section{La historia dinástica de Machaquilá según el registro epigráfico}

Las fuentes para la historia política de Machaquilá proceden de su propio corpus jeroglífico de textos y de los de otros yacimientos que en algún momento mencionan personajes o eventos relacionados con el sitio. Entre las menciones foráneas podemos destacar las de Cancuén (Estela 1, Panel de los 160 Glifos, Panel 3, Escalera Jeroglífica 1, Marcador de Juego de Pelota 2), Dos Pilas (Escalera Jeroglífica 2, Escalera Jeroglífica 4, Panel 7), Tres Islas (estelas 1 y 2), así como un vaso de procedencia desconocida, de estilo Holmul.

Diferentes aspectos de la historia epigráfica de Machaquilá han sido tratados en diversas ocasiones, como objeto específico de estudio o comentados indirectamente en la discusión de asuntos relacionados. Además del importante trabajo inicial de Graham (1967: 51-99) identificando el Glifo Emblema, estableciendo la cronología epigráfica del sitio y reconociendo el nombre y títulos de algunos gobernantes, cabe destacar los primeros trabajos de Federico Fahsen $(1983 ; 1984)$, quien refina la primera secuencia dinástica de Clásico Tardío del sitio de, entonces, seis gobernantes conocidos. A estos trabajos hay que añadir los de Riese (1984, 1988a, 1988b), Reents-Budet (1991), Mathews y Willey (1991), Houston (1992, 1993), Stuart y Houston (1994), Schele y Grube (1994; 1995), Fahsen y Demarest (2001), Fahsen (2002), Fahsen y Jackson (2002), Boot (2002, 2005: 49-61), Guenter (2002; 2003), Fahsen et al. (2003; Fahsen, Demarest y Luin 2003), Demarest y Fahsen (2003), Tomasic y Fahsen (2004), Kistler (2004), Zender (2004, 2005), Zender y Skidmore (2004), Demarest et al. (2006), el extenso y muy completo trabajo de Just (2006) -que dedica una buena parte de su tesis doctoral al estudio de la escultura monumental y los textos de Machaquilá-y, en el contexto del proyecto, Iglesias y Lacadena (2003), Ciudad et al. (2004), Lacadena e Iglesias (2005), Ciudad y Lacadena (2006), Lacadena (2005, 2006), y Ciudad et al. (2010) -un sumario con la secuencia dinástica de Machaquilá actualizada se ofrece en el Apéndice 2-. 


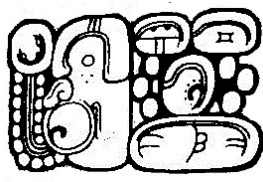

a

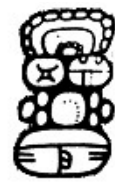

$\mathrm{b}$

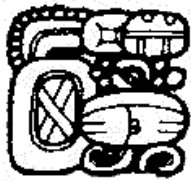

c

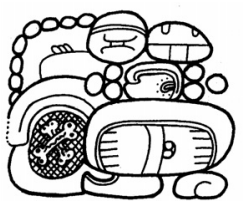

d

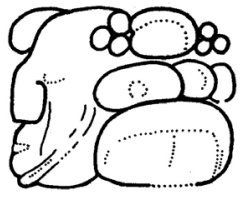

e

Figura 2: El Glifo Emblema de Machaquilá: a= Machaquilá, Estela 11, A5; b= Machaquilá, Estela 3, G1; c= Machaquilá, Estela 6, B3; d= Machaquilá, Estructura 4, Frag. E-86; e= Machaquilá, Frag. E92. (a-c, según dibujos de I. Graham [1967: figs. 63, 49 y 55]; d-e, según dibujos de A. Lacadena).

\subsection{Machaquilá, sede de una dinastía real}

El Glifo Emblema de Machaquilá aparece ya identificado en la obra de Graham (1967), y discutido en Fahsen (1983; 1984). Es un Glifo Emblema completo, en el sentido de que, además del título ajaw «señor, rey»-escrito en el sitio como AJAW o AJAW-wa-, incorpora en época tardía el adjetivo k'uhul «sagrado»-escrito normalmente en su forma abreviada como K'UH, $k^{\prime} u h[u l]$ (Figura 2a-d)-. Este último logograma presenta interesantes variantes gráficas poco usuales, como las de la Estela 6 o la Banca Jeroglífica (Figura 2c y d). Desgraciadamente, el nombre del reino de Machaquilá consta de un signo aún sin descifrar - una variante del signo T174-, lo que impide tener una lectura completa. Sabemos que el nombre del reino termina en /s/ por la presencia recurrente en posición final del fonograma T216 su. En la Estructura 4 de la Plaza F se encontró un gentilicio femenino basado en el topónimo de Machaquilá, escrito como IX-174-ti-su, incluyendo un signo ti entre T174 y su (Figura 2e), dato que habrá que tener en cuenta a la hora de leer el compuesto. La importancia de la identificación del Glifo Emblema en Machaquilá es grande, porque permite asignar a Machaquilá la categoría política de reino en el paisaje político de las Tierras Bajas mayas del Periodo Clásico. El reino - ajawlel-, con el rey -ajaw-a la cabeza, es la entidad política superior dentro de la organización política maya (Mathews 1991; vid. Lacadena y Ciudad 1998). El glifo Emblema de Machaquilá se documenta al menos desde el 475 d.C. en las estelas 1 y 2 de Tres Islas -aunque en la Estela 1 hay eventos mencionados desde el 396 d.C.--, hasta mediados del siglo IX en Machaquilá (la última referencia segura datada es el 840 d.C.).

Dos gobernantes del siglo IX, Ochk'in Kalo'mte' y Siyaj K' in Chaahk II se asociaron al título kalo 'mte', el primero como parte de su nombre, y el segundo como título. El título kalo 'mte', aún de etimología incierta, fue ostentado sólo por algunos de los más importantes reyes sagrados mayas del Periodo Clásico, normalmente restringido a aquellos gobernantes que tienen otros $k^{\prime} u h u l$ ajaw subordinados (Martin y Grube 2000; Houston e Inomata 2009: 141). Otros títulos recurrentes ostentados por los gobernantes de Machaquilá son los de b'a-ka-b'a, b'aah kab' «príncipe de la tierra», en ocasiones asociado al número waxak winik «veintiocho», lo que es frecuente en época tardía sobre todo en la región oriental de Tierras Bajas, y u-CHAN-na, uchan «el guardián de», que señala la relación entre el vencedor y su vencido, haciendo alusión siempre a un contexto bélico o guerrero. 


\subsection{La época temprana de la dinastía de Machaquilá (ca. 200 d.C. - ca. 670 d.C.)}

La época temprana de Machaquilá es una época llena de luces y sombras, en la que datos de extrema importancia desde el punto de vista político contrastan con la escasez de fuentes y la ausencia de registro material. Si algo caracteriza a los datos sobre la historia temprana de Machaquilá es, primero, su procedencia, de textos de fuera del propio yacimiento arqueológico; y, segundo, la presencia de ciertos elementos que serán luego recurrentes en la historia tardía del sitio.

La primera mención a Machaquilá aparece en un vaso de cerámica de Clásico Tardío de estilo Holmul, K633, que representa una escena mitológica con tres personajes ataviados como el dios el maíz (Figura 3). Los tres personajes están bailando en compañía de sendos enanos y portando a la espalda animales mitológicos. Lo interesante del vaso es que los tres personajes están asociados al nombre de tres lugares: ka-KAN, Kan [u'l] (Calakmul), MUT, Mut [u'l] (Tikal), y T174-su (Machaquilá) (Reents-Budet 1991). Kanu'l (con su primera capital en Dzibanché y luego en Calakmul) y Mutu'l (con su capital en el actual sitio arqueológico de Tikal) fueron los dos reinos más poderosos de las Tierras Bajas mayas durante el Periodo Clásico. La historia de las Tierras Bajas mayas en este periodo no puede entenderse sin el persistente antagonismo que enfrentó a estos dos colosos, quienes rivalizaron en la construcción de amplias hegemonías políticas (Martin y Grube 1994; 1995; 2000). No será la única vez que la historia de Machaquilá se entrecruce con la de estos dos gigantes. En cualquier caso, lo que nos interesa de este vaso es que aparece el nombre del reino de Machaquilá asociado a un evento mítico, lo que nos habla de la importancia que tuvo Machaquilá en la geografía sagrada de los mayas clásicos. Sitios destacados de esta manera en la mitología maya suelen ser lugares de gran profundidad temporal histórica que hunden las raíces del origen de sus dinastías en el Preclásico Tardío, como los propios Calakmul y Tikal.

La antigüedad de la entidad política de Machaquilá sugerida por su inclusión en la escena mítica de K633 queda confirmada por las menciones -ahora sí, históricas-que se hacen del reino en textos del Clásico Temprano. Estas menciones se producen en el sitio de Tres Islas, situado cerca de la confluencia del río Machaquilá-Santa Amelia

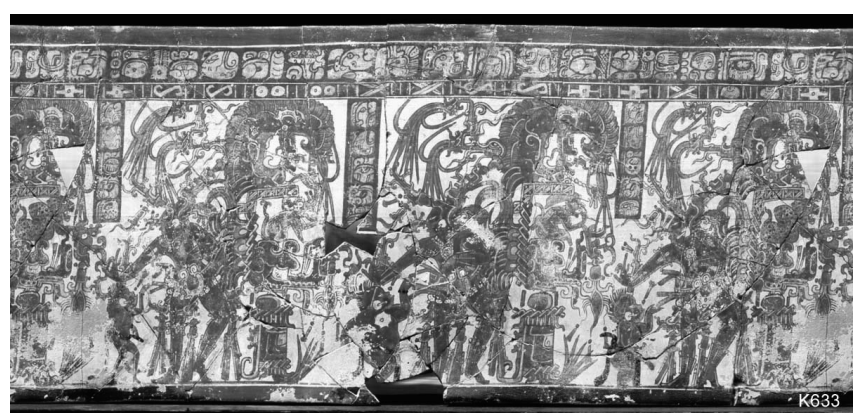

a

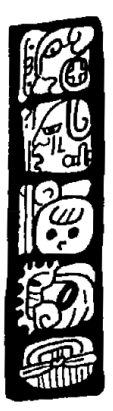

b

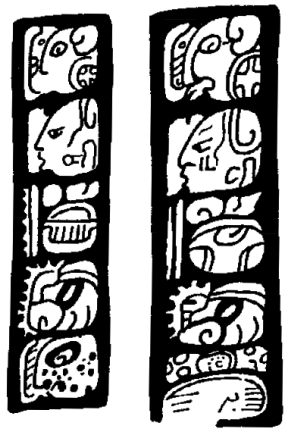

Figura 3: Machaquilá en el mito: a= Vaso de estilo Holmul (K633) (según Reents-Budet 1994: 63); $b=$ los textos de K633 en columna (en la columna de la derecha, el Glifo Emblema de Machaquilá) (según dibujo de D. Reents-Budet [1991: Fig. 7]). 

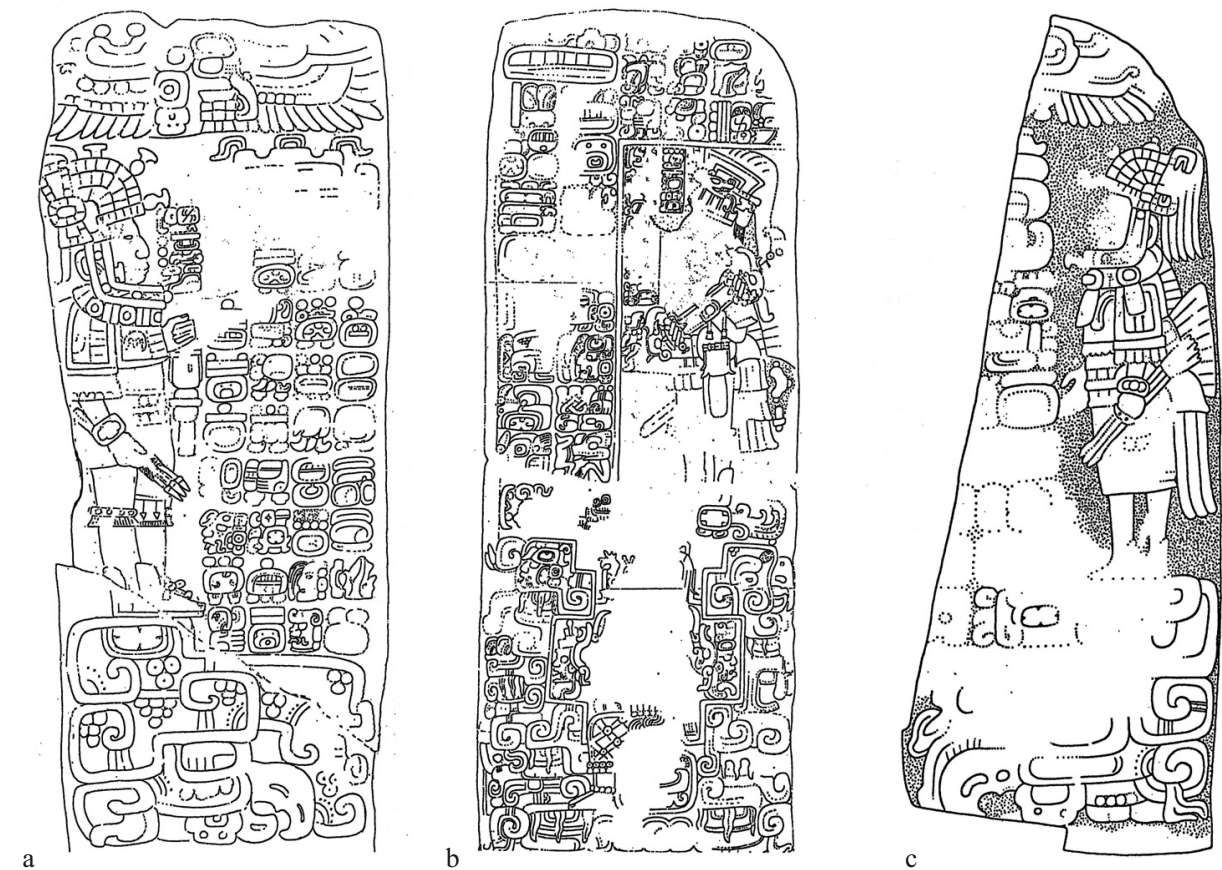

Figura 4: Las estelas de Tres Islas: $\mathrm{a}=$ Estela 1; $\mathrm{b}=$ Estela 2; $\mathrm{c}=$ Estela 3 (según bocetos de I. Graham).

con el río Pasión (Houston 1993; Schele y Grube 1994; Fahsen y Demarest 2001; Fahsen y Jackson 2002; Kistler 2004: 3; Tomasic y Fahsen 2004; Tomasic et al. 2005; Just 2006: 39 y ss.; Demarest et al. 2006). En Tres Islas se encontraron tres estelas alineadas norte-sur sobre una plataforma, con un altar asociado en el oeste (Figura 4). Las estelas mencionan varias fechas entre 8.18.0.0.0 y 9.2.0.0.0 (entre el 396 y el 475 d.C.). Esta última fecha, el final del katún 9.2.0.0.0 en 475 d.C. es la fecha de dedicación de los monumentos. Estos monumentos de Tres Islas son llamativos en todos los sentidos: iconográficamente, cada uno representa en su frente un personaje, los de las estelas 1 y 3 flanqueando al personaje central, al cual miran. Los tres personajes llevan tocados de estilo teotihuacano; los personajes de las estelas de los extremos, van vestidos de guerreros y portan yelmo y haces de dardos característicos de Teotihuacan en una mano, y el de la Estela 1 una bolsa de copal en la otra, característica también de Teotihuacan. Las inscripciones de las estelas - de las que sólo existen bocetos por lo que la comprensión de su contenido es aún incompleto- mencionan varios personajes, entre los que se puede reconocer a un rey de Machaquilá, otro de Cancuén y a un tercer personaje mencionado con el título de chan tz'akb'uul Wite'naah ajaw «cuarto sucesor, rey de Wite'naah», que es el personaje representado en la Estela 2, del centro, portando una barra ceremonial. Wite'naah, un topónimo, y chan tz'akb 'uul «cuarto sucesor», un título, son expresiones que aparecen en el Marcador de Juego de Pelota de Mundo Perdido y la Estela 31 de Tikal en relación a unos personajes y unos acontecimientos que algunos investigadores han interpretado como la llegada de un 
contingente de teotihuacanos a Tikal en 378 d.C. y la implantación y extensión de su dominio sobre ciertas zonas de Petén en las décadas siguientes (Stuart 2000; Martin y Grube 2000; Nielsen 2003; Fash et al. 2009).

Si es llamativa esta primera mención a Machaquilá asociada a elementos iconográficos teotihuacanos y a referencias epigráficas de personajes vinculados en otras áreas de Petén a acontecimientos cruciales desde el punto de vista político, llamativa es también la ubicación de los monumentos, en un sitio al que difícilmente se puede dar categoría de centro urbano, ni siquiera pequeño (Tomasic y Fahsen 2004; Tomasic et al. 2005), y que, evidentemente, no puede ser la capital temprana ni de Cancuén ni de Machaquilá. Dada la habitual asociación de las estelas a espacios públicos urbanos de significación política, sorprende entonces la erección de este conjunto de monumentos de Tres Islas casi en medio de la nada, a orillas del río Pasión. La interpretación del conjunto es difícil. A la espera de nuevas evidencias (y de una mejor reproducción de las inscripciones de los monumentos), sólo podemos hipotetizar sobre la función de estas estelas y las razones que llevaron a su erección. Uno de los escenarios sugeridos es una confederación temprana entre los reinos de Machaquilá y Cancuén (Fahsen y Demarest 2001: 1003; Demarest y Fahsen 2003: 160); otro escenario propuesto es que en esos tiempos tempranos los gobernantes de Tres Islas ostentaran a la vez los glifos emblema de Machaquilá y Cancuén y que sólo con posterioridad, tras una posible ruptura entre linajes rivales, se escindieran los reinos de Machaquilá y Cancuén (Just 2006: 39). Un tercer escenario que podemos sugerir, atendiendo al tipo de asentamiento de Tres Islas - que no es un centro urbano, ni Tres Islas en realidad tiene categoría de reino-, la iconografía teotihuacana y la mención del chan tz'akb'uul Wite'naah ajaw «cuarto sucesor, rey de Wite'naah», es que los monumentos erigidos de Tres Islas representen la verificación (o la memoria de ello) de un acuerdo territorial fronterizo entre Machaquilá y Cancuén, sancionado por una autoridad superior, entonces representada por el nuevo orden teotihuacano.

La mención al rey de Machaquilá, el primero de cuyo nombre se tiene noticia, se hace al menos en dos ocasiones, en las estelas 1 y 2 , en la primera mencionado como el personaje que erige el monumento. Su nombre ha sido leído de varias maneras, como Yax Te' K'inich (Tomasic y Fahsen 2004), como Yax...K'inich (Ciudad y Lacadena 2006) y como Yax Tzu' Chaahk (García Barrios 2009). Pero no es el primer gobernante de Machaquilá. Un título que ostenta en la Estela 2 lo describe como «decimonoveno sucesor» de su dinastía (Schele y Grube 1994; Fahsen y Demarest 2001; Fahsen y Jackson 2002). Si aplicamos una media conservadora de 15 años por reinado, tenemos que el fundador dinástico de Machaquilá debió reinar unos doscientos setenta años antes, hacia finales del siglo II d.C. Esta fecha de finales del Periodo Preclásico no contradice las fechas de fundación alegadas por otras dinastías reales clásicas en el área maya, ocurridas a finales del Preclásico Tardío, en los dos primeros siglos de Nuestra Era. En todo caso, resulta importante resaltar las siguientes cuestiones: primero, la existencia de Machaquilá como reino desde el comienzo mismo del Periodo Clásico; segundo, la implicación de Machaquilá desde tiempos tempranos en asuntos políticos más allá de sus fronteras; y tercero, la especial relación política y geográfica del reino de Machaquilá y el reino de Cancuén desde antiguo, que en esa época debían estar territorialmente más cerca uno de otro. 
Pero la evidencia de los textos de Tres Islas sobre la existencia de reyes de Machaquilá de Clásico Temprano contrasta radicalmente con la interpretación de los materiales arqueológicos excavados en el propio yacimiento de Machaquilá, que sugieren una fundación y una ocupación de la ciudad mucho más tarde, en el Clásico Tardío (vid. Ciudad y Lacadena 2006). Esto lleva a pensar, lógicamente, que la capital del reino de Machaquilá en el Clásico Temprano estuvo en lugar distinto al de la ubicación actual. Se ha especulado mucho acerca de dónde estuvo esta primera capital. Con la información epigráfica disponible, en Ciudad y Lacadena (ibid.) se planteó la posibilidad de que la ciudad estuviera originalmente más cerca del Río Pasión, al alcance de la intervenciones militares de Dos Pilas en tiempos de Bajlaj Chan K'awiil -en 664 d.C. un personaje de Machaquilá es capturado por Dos Pilas- aunque necesariamente no muy alejado de Tres Islas, sitio que ancla geográficamente la capital temprana del reino de Machaquilá a la zona. Se sugirió entonces algún lugar hacia el oeste de la ubicación de Clásico Tardío, en las cuencas del curso bajo de los ríos Machaquilá-Santa Amelia o Poxté-San Juan, más cercano a sus desembocaduras en el Río Pasión, con los límites de Seibal o El Cedral al norte, y Tres Islas al sur. Resulta sumamente interesante que recientes exploraciones por parte de miembros del Proyecto Cancuén en la región de Tres Islas han señalado la existencia de nuevos yacimientos en el curso bajo del mismo río Machaquilá, entre Machaquilá y Tres Islas (Tomasic y Fahsen 2004; Tomasic et al. 2005). Es posible que uno de esos yacimientos, una vez excavados, proporcione la identificación de la capital perdida.

Nada más sabemos de Machaquilá en esta primera época temprana de su dinastía real. Confiamos en que el hallazgo de la primera capital pueda proporcionar información sobre esos primeros diecinueve reyes y sus sucesores, sobre cómo se realizó la introducción de elementos teotihuacanos en la región, sobre la naturaleza de las relaciones con el reino de Cancuén y sobre los acontecimientos que llevaron a la dinastía real a abandonar su capital y a desplazarse hasta el actual sitio de Machaquilá a comienzos del Clásico Tardío.

\subsection{La refundación de Machaquilá (ca. 670->776 d.C.)}

No cabe duda de que el gran acontecimiento que inaugura este periodo es el abandono de la capital del Clásico Temprano y la fundación de un nuevo asentamiento. En Ciudad y Lacadena (2006) se ha especulado sobre las razones que compelieron a los gobernantes de Machaquilá a trasladar su sede. La Escalera jeroglífica 2 de Dos Pilas relata que en 664 d.C., un personaje de Machaquilá nombrado con el título genérico de Tajal Mo' fue capturado por el rey Bajlaj Chan K'awiil de Dos Pilas (Houston y Mathews 1985: 10; Houston 1993; Martin y Grube 2000: 56; Boot 2002; Guenter 2002, $2003)^{1}$. La victoria militar de Dos Pilas sobre Machaquilá no fue una simple confron-

\footnotetext{
${ }^{1}$ El texto (Dos Pilas, Escalera jeroglífica 2, Escalón II, C1-F2) dice: 9-[KAB'AN] 5-K'AN-JAL-wa chuka-ja TAJ-MO'-o AJ-T174-\# u-B'AK-[ki] b'a-la-ja CHAN-na K'AWIL-la K'UH-MUT-la-AJAW-wa b'a-ka-b'a, $9 \mathrm{Kab}$ 'an $5 \mathrm{~K}$ 'anjal[a']w chu[h]kaj Taj[al] Mo'aj«Machaquilá» ub'aak B'a[j]laj Chan K'awiil K'uh[ul] Mut[u']l Ajaw, B'a[ah] Kab' «(en) 9 Kab'an 5 Pop fue capturado Tajal Mo', el de «Machaquilá», el cautivo de B'ajlaj Chan K'awiil, rey sagrado de Mutu'l, Príncipe de la Tierra».
} 
tación local entre dos estados vecinos. La victoria se produjo en el contexto de la larga guerra que enfrentó a Calakmul (Kanu'l) y Tikal (Mutu'l) a mediados del siglo VII por la supremacía política en el área maya y en la que la región del río Pasión fue un escenario de batalla más (vid. Houston 1992, 1993; Martin y Grube 2000; Boot 2002; Guenter 2002, 2003; Fahsen et al. 2003). En esta guerra, Machaquilá tomó posiblemente partido por Tikal, como lo hizo también inicialmente Dos Pilas. Los reveses militares sufridos por Tikal y sus aliados frente a Calakmul (Calakmul ataca Dos Pilas en 650 d.C. y la propia ciudad de Tikal en 657 d.C. forzando el exilio temporal de su rey), el cada vez más firme control de Calakmul de la región del río Pasión -los reyes de Cancuén rinden pleitesía a Calakmul desde 656 d.C. accediendo al poder bajo sus auspicios- y la deserción final de Dos Pilas del bando de Tikal, aliándose con Calakmul desequilibrando la balanza e iniciando una verdadera depredación de la zona, posiblemente dejaron a Machaquilá aislada y a merced de la agresiva Dos Pilas por el norte, al tiempo que amenazada por Cancuén desde el sur. La derrota y captura de Tajal Mo' en 664 d.C. fue quizá una advertencia o incluso el detonante. La derrota final de Tikal en 679 d.C. a manos de B'ajlaj Chan K'awiil vino a confirmar la supremacía militar de Dos Pilas y el papel predominante que iba a desempeñar en el área durante las siguientes décadas. El abandono de la capital temprana y la elección de un nuevo emplazamiento pudo ser, entonces, la estrategia adoptada por el gobernante de Machaquilá para poner a salvo a la dinastía y la propia supervivencia del reino, alejando su sede de la zona de conflicto, retirándose del río Pasión hacia el interior.

Siyaj K'in Chaahk I es el primer gobernante mencionado en una inscripción de la nueva capital, y quizá el artífice de su traslado. En realidad, sólo conocemos a este gobernante por la mención que hace de él y de su esposa -Ix Yax Paach K'uk', una mujer de rango ajk'uhu'n- su hijo y sucesor «Etz'nab'» Chaahk, en el largo texto de la Estela 11 (Figura 5) ${ }^{2}$. Las fechas de su reinado son inciertas, debiendo ubicarse en

${ }^{2}$ La esposa de Siyaj K'in Chaahk I es una noble ixajkuhu'n, de un linaje sacerdotal posiblemente perteneciente al propio reino de Machaquilá, en vez de una ixajaw, una princesa de sangre real de algún reino vecino, 
o antes de 9.14.0.0.0, 711 d.C., dependiendo de a quién asignemos la erección de la Estela 13, el primer monumento datado del sitio, que desafortunadamente no conserva referencias a nombres personales, y está separada veinte o treinta años de la siguiente mención calendárica. Dado el patrón espacial y cronológico de las estelas de la Plaza A, especulamos con la posibilidad de que sean monumentos suyos las estelas 14, 15 y 16 ubicadas en el este y asociadas a la Estructura 16, aunque desgraciadamente eran planas o se encuentran tan erosionadas que resultan ilegibles. Con independencia de a qué gobernante asignemos la Estela 13, si a Siyaj K'in Chaahk I o a su sucesor «Etz'nab'» Chaahk, lo cierto es que la fecha de 711 d.C. que conmemora es una fecha ante quem para la fundación de la nueva capital, que tuvo que haberse producido en el $k^{\prime}$ atun o dos k'atunes anteriores.

«Etz'nab'» Chaahk ${ }^{3}$ es el primer gobernante del nuevo asentamiento asociado con claridad a fechas absolutas. Monumentos comisionados en su reinado son las estelas 10, 11 y 12 ( vid. Figura 5), asociadas a la Estructura 17 de la Plaza A. A la erosionada Estela 10 se le ha asignado la fecha de 9.15.0.0.0, 731 d.C. (vid. Graham 1967: 77, 79; Fahsen 1984: 98, Tabla 1; Boot 2005: 49), aunque es problemática4 ${ }^{4}$ Por su parte, las

como suele ser común en los matrimonios de los gobernantes, y es confirmado por las otras dos ocasiones en que se mencionan esposas de reyes de Machaquilá (la esposa de Jun Tzak Tok' es ixajaw -el nombre del reino está aún indescifrado-, lo mismo que la presunta esposa de «Escorpión» Ti' Chaahk, una ixajaw del reino de Mahk). El bajo rango de la esposa de Siyaj K'in Chaahk I podría ser reflejo de las dificultades iniciales de esta nueva etapa en la recién fundada capital, con la accesión final al poder de un candidato secundario, una vez desaparecidos los candidatos principales en los tiempos turbulentos en los que se produce el traslado.

${ }^{3}$ El nombre de este gobernante está escrito en la Estela 11 (B4) como YAX-4-PAS AJAW-wa «ETZ'NAB'» CHAK-ki, lo que daría el nombre completo de Yax Chan Pas Ajaw «Etz'nab'» Chaahk, o incluso Yax Chan Pas[aj] Ajaw «Etz'nab'» Chaahk -Just (2006) se refiere a él como Yax 4? Pas Etz'nab Chaak-. Si toda esta secuencia es un único nombre representando un apelativo del dios Chaahk, éste quizá podría traducirse como ««Etz'nab'» Chaahk es el señor de los primeros cuatro amaneceres». La primera parte del nombre, sin embargo, podría ser problemática, en lo que se refiere al orden de lectura de los signos. Así, por ejemplo, Boot (2005: 50) lee esta primera parte como Yax Pasah Chan Ahaw, con chan «cuatro»-actuando en rebus para chan «cielo»-, lo que lo haría cercano a otros apelativos conocidos, como Yax Pasaj Chan Yop Aat, de Copán («Yop Aat es el primer amanecer en el cielo»). En el caso de Machaquilá, ajaw «rey, señor», sustituiría el nombre de la deidad Yop Aat. Otra alternativa sería considerar que chan «cuatro» debe leerse en primer lugar, resultando Chan Yax Pas Ajaw. Recientemente, Houston y Martin (2010) han mostrado ejemplos en los que CHAN «cuatro» sustituye a la expresión completa $u$-cha(') $n$ «el guardián de», normalmente escrita como u-CHAN (chan «serpiente»). Aunque aparentemente en este ejemplo de Machaquilá YAX se lee antes que CHAN «cuatro», quizá los problemas de composición causados por la escritura de tres palabras en un mismo bloque jeroglífico, fueron resueltos por el escriba alterando el orden de colocación de los signos. El resultado, entonces, sería, [u]chan Yax Pas ajaw, «Etz'nab'» Chaahk, donde «Etz'nab'» Chaahk sería el propio nombre del rey de Machaquilá y [u]chan Yax Pas ajaw el título «el guardián del señor de Yax Pas». En este caso, Yax Pas o Yax Pas[aj] sería, entonces, un topónimo. De ser así, el gobernante de Machaquilá iría precedido y seguido por dos título de «guardián»: «guardián del señor de Yax Pas, «Etz'nab'» Chaahk, guardián del señor de Ik'». Este posible ejemplo de Machaquilá de CHAN por [u]chan, fechado en 741 d.C., no queda lejos del periodo de $769-$ 799 d.C. en que se documentan los ejemplos señalados por Houston y Martin. Dado lo problemático del nombre, me referiré en lo sucesivo a este personaje como «Etz'nab'» Chaahk.

${ }^{4} \mathrm{La}$ información calendárica de la Estela 10 se encuentra en los bloques A1-A5 que abren la inscripción, aunque se encuentran muy erosionados. La propuesta de Graham $(1967: 77,79)$ de fechar la Estela 10 en 9.15.0.0.0 4 Ajaw 13 Yax se basa en la identificación del motivo de la base sobre el que se encuentra el gobernante con un cartucho de tzolk'in gigante, presumiblemente Ajaw, y la cabeza del interior con la del dios del Sol, funcionando como variante de cabeza del número cuatro. Sin embargo, el pretendido cartucho es en realidad el icono del cuadrilobulado, y la cabeza emergente es la del dios Chaahk. Esto no contradice la atribución de la estela a «Etz'nab'» Chaahk, cuyo nominal se encuentra posiblemente en el bloque B7. 
estelas 11 y 12 registran, respectivamente, las fechas de 9.15.10.0.0, 741 d.C., y 9.16.10.0.0, 761 d.C. (Graham 1967: 85, 86). El reinado de «Etz'nab'» Chaahk pudo durar, por tanto, cincuenta años (si le atribuimos la fecha de 711 d.C. de la Estela 13), treinta (si aceptamos la fecha propuesta de 731 d.C. de la Estela 10 -vid. Notas 4 y 5-), o cuando menos un mínimo de veinte, un lapso de tiempo tampoco desdeñable. La estabilidad política, social y económica que proporciona un reinado largo fue sin duda una ventaja para la consolidación de la dinastía y la población en el nuevo asentamiento y su desarrollo urbano.

El nuevo emplazamiento elegido para edificar la ciudad fue un éxito. Fue también un acierto el posible cambio de alineación política del reino, que terminó definitivamente de abandonar el bando de Tikal, que ya, tras el control de Calakmul y sus aliados de la zona, quedaba como un poder más lejano. El cambio de alineación política quizá pueda confirmarse en el título guerrero que ostenta «Etz'nab'» Chaahk en la Estela 11, u-CHANna IK'-AJAW, uchan I $k$ ' ajaw «el guardián del rey de Ik'». Si Ik' se refiere a Ik'a', el reino que tuvo su capital en el Motul de San José, en el Petén Itzá, Machaquilá habría atacado entonces a uno de los aliados tradicionales de Tikal. La propia Dos Pilas captura un gobernante de Ik' en el 745 d.C. (Houston

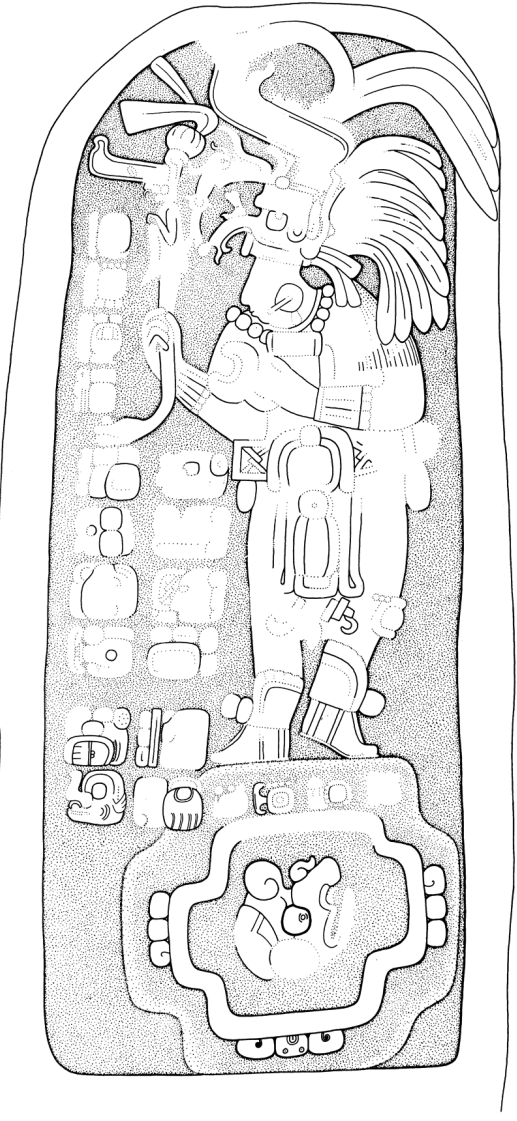

Figura 6: Machaquilá, Estela 10 (según dibujo de I. Graham [1967: Fig. 61]). 1993), no mucho después de la fecha de 741 d.C. de la Estela 11 de Machaquilá.

Durante el mandato de «Etz'nab'» Chaahk la ciudad va a crecer y a desarrollarse en torno a ejes bien definidos y planificados. Uno de los elementos urbanos más estables en el tiempo va a ser la Plaza A, que se diseña como un espacio de acceso restringido y que, en lo sucesivo (aunque quizá ya desde los tiempos de su padre, Siyaj K'in Chaahk I), va a alojar estructuras piramidales alineadas al norte y este del espacio central de la plaza, posiblemente las tumbas reales de la dinastía -también sugerido por Just (2006)-, en cuyos frentes se van a ubicar las estelas de los sucesivos gobernantes. Quizá ya entonces el centro de la plaza está ocupado por la primera versión de una construcción especial, un recinto rehundido en forma de cuadrilóbulo, que tendrá su primera representación iconográfica en la base de la Estela 10, como un icono cuadrilobulado en cuyo interior se encuentra la cabeza vuelta hacia arriba, posiblemente emergiendo, del dios Chaahk (Houston et al. 2005: 5-7; Just 2006: 46; García Barrios 2009: 244-249) (Figura 6). Desde los inicios de la nueva Machaquilá y hasta la desa- 


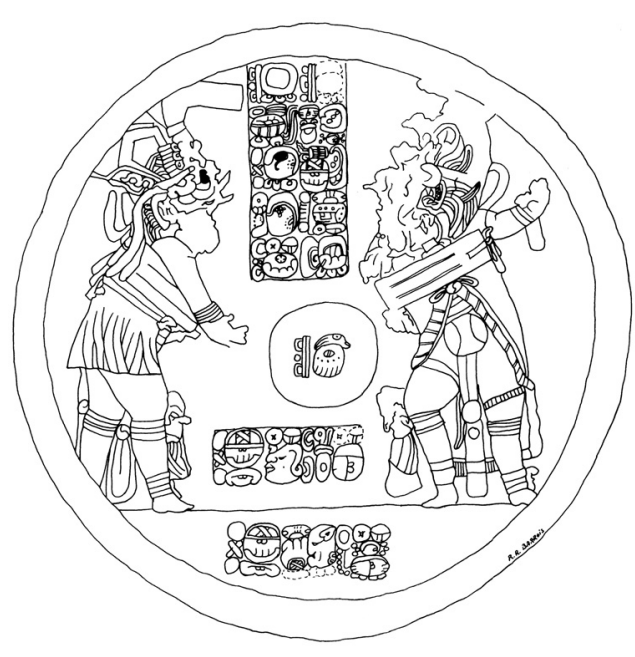

Figura 7: Cancuén, Marcador de Juego de Pelota 2 (según dibujo de R. Barrois).

parición de su dinastía real más de un siglo más tarde, la Plaza A y el recinto cuadrilobulado jugarán un papel central en los rituales dinásticos vinculados a las conmemoraciones calendáricas y el culto a las deidades del agua y a los antepasados reales.

La sucesión de «Etz'nab'» Chaahk no está clara, como tampoco los acontecimientos que se desencadenaron en el último cuarto el siglo VIII y que culminaron en un enfrentamiento armado con Cancuén, teniendo como resultado la captura de un rey de Machaquilá. Sabemos que un nuevo gobernante de Machaquilá erige la Estela 18 en el eje este-oeste de la Plaza C, asociada a las fechas 775 y 776 d.C. (Fahsen 1984, 1984; Riese 1988b), y quizá también la Estela 19 (que no conserva fecha legible, pero es muy parecida en estilo), originalmente alineadas con la Estela 175, ubicadas en el mismo eje de la Plaza C. Otro gobernante -quizá el mismo de la Estela 18-aparece mencionado en el Marcador de Juego de Pelota 2 de Cancuén (Figura 7), como cautivo de un personaje subordinado al rey Tajal Chan $\mathrm{Ahk}^{6}$ de este último reino (Zender y Skidmore 2004; Zender 2004; Demarest et al. 2006). Sólo sabemos el nombre del cautivo, Chak ...B'ahlam, que era «rey sagrado de Machaquilá», y que la captura se

\footnotetext{
${ }^{5}$ La datación de la Estela 17 es sumamente problemática. A partir de las fotografías de Graham (1967: Fig. 70), Just (2006: 70-72) propone asignar la fecha de 9.18.5.0.0 4 Ajaw 13 Keh, 795 d.C., a la Estela 17, basado en la posible Rueda Calendárica 4-? 13-? que ocupan los bloques $7^{\circ}$ y $8^{\circ}$ del texto (B3-C3), tras un posible Glifo Introductorio de Serie Inicial (A1) y cinco órdenes de Cuenta Larga (B1-C2), y el estilo del monumento. Otra alternativa sería 9.15.0.0.0 4 Ajaw 13 Yax, 731 d.C., que colocaría la estela dentro del reinado de «Etz'nab'» Chaahk. Aunque con el incompleto registro disponible del monumento todo es especulación, si en B4C4 se encontrara un verbo y su objeto, en B5-C5 esperaríamos encontrar el nombre o títulos del gobernante. Si esto fuera así, el perfil de los signos que se adivina, con un primer bloque con aspecto de $t z o l k$ 'in sin coeficiente y un segundo bloque compuesto por un signo de cabeza y un sufijo, podría bien corresponder al nombre «ETZ'NAB'» CHAK-ki, «Etz'nab'» Chaahk. Interesantemente, Graham (1967: 90-92) apunta ciertas semejanzas estilísticas entre la Estela 17 y la Estela 10, que se puede atribuir a este gobernante (su nombre estaría escrito en el bloque B7).

${ }^{6} \mathrm{Al}$ menos en dos ocasiones la parte final del nombre de este rey se escribe como a-ku, que sugiere una lectura $A h k u$ 'l o $A h k u l-\mathbf{a}-\mathbf{k u}$ es abreviatura de a-ku-la y a-ku-lu-, en vez de simplemente $A h k$. Con todo, por ser más conocido este gobernante como Tajal Chan Ahk en la literatura, se usará aquí esta versión de su nombre.
} 
tuvo que producir en algún momento anterior al año 795 d.C. Chak ... B'ahlam es, por tanto, candidato a ser el gobernante que dedica las estelas 18 y 19 de Machaquilá ${ }^{8}$.

\subsection{El dominio de Cancuén (ca. >776? - 799 d.C.)}

La captura de Chak ... B'ahlam puso a Machaquilá bajo el dominio de Cancuén. No se trató sólo de una simple subordinación política típica del modelo hegemónico político maya, sino que el rey de Cancuén, Tajal Chan Ahk, se convirtió también, formalmente, en el rey de Machaquilá, uniendo los Glifos Emblemas de ambos reinos en sus inscripciones. Tajal Chan Ahk nació en 742 d.C. y accedió al poder en 757 d.C. cuando era un adolescente. Reinó en Cancuén hasta el 799 d.C., tras un largo gobierno de cuarenta y dos años, siendo el responsable del apogeo final del reino de Cancuén en la región y el artífice del esplendor arquitectónico de su última capital (Fahsen y Demarest 2001; Fahsen y Jackson 2002; Guenter 2003; Fahsen et al. 2003; Kistler 2004; Zender y Skidmore 2004; Demarest et al. 2006).

Dada la dinámica de la sucesión de títulos reales entre los antiguos mayas clásicos, por la que el acceso a dichos títulos no podía hacerse a través de la conquista sino sólo mediante la herencia de sangre, la utilización del título «rey de Machaquilá» por Tajal Chan Ahk de Cancuén tiene dos implicaciones: la primera es la muerte del rey Chak ... B'ahlam de Machaquilá; la segunda es la evidencia de que Tajal Chan Ahk estaba vinculado por lazos de sangre con la familia real de Machaquilá. Es la existencia de esta vinculación de sangre la que permitió a Tajal Chan Ahk de Cancuén postularse como candidato al trono de Machaquilá tras la desaparición del último rey. La vinculación familiar entre ambas casas reinantes, la de Machaquilá y Cancuén, no debe sorprendernos. Son muchas las evidencias que tenemos acerca de las uniones matrimoniales entre miembros de casas reinantes distintas como una de las estrategias fundamentales de alianza de las élites mayas del Periodo Clásico. La existencia de lazos familiares derivados de matrimonios de esta índole entre las casas reales de Machaquilá y Cancuén es verosímil, dada su condición de reinos vecinos y la larga historia común que arranca ya desde el Periodo Clásico Temprano, con la mención de gobernantes de ambos reinos en los textos de Tres Islas.

No sabemos la duración precisa de la unión de ambas coronas en la persona de Tajal Chan Ahk de Cancuén, que depende de cuándo se produjo la captura y muerte de Chak ... B'ahlam de Machaquilá. Por un lado, tenemos las fechas asociadas a las menciones dobles de los Glifos Emblema en Cancuén que son tardías en el reinado de Tajal Chan Ahk, de los años 795 (Panel 3) y 799 d.C. (Panel de los 160 Glifos). Esto concordaría con la fecha de 9.18.5.0.0, 795 d.C. propuesta por Just (vid. Nota 5) para la Estela 17 de Machaquilá, quien sugiere ese mismo año de 795 d.C. para la

\footnotetext{
${ }^{7}$ La propia fecha del Marcador de Juego de Pelota 2 de Cancuén que menciona al guardián de Chak ... B'ahlam, expresada con Rueda Calendárica, está muy erosionada. Fahsen (en Zender y Skidmore 2004) la reconstruye como 9.18.5.5.19 6 Kawak 12 Kumk'uh, en 796 d.C., aunque el día del Tzolk'in podría ser Hix. En cualquier caso, el marcador de Juego de Pelota 1 al que se asocia lleva la fecha de 9.18.5.0.0, que es posiblemente la fecha de dedicación de conjunto de los marcadores originales.

${ }^{8}$ Un nuevo dibujo del bloque E3 de la Estela 18 (conservada en una colección privada en Europa), donde se encuentra el nombre del gobernante, terminaría de dirimir esta cuestión.
} 


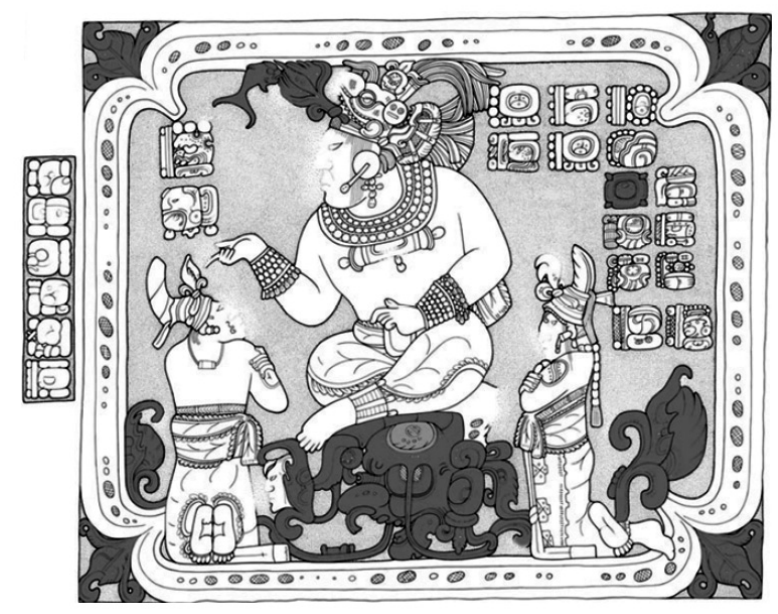

Figura 8: Cancuén, Panel 3 (según dibujo de F. Luin, en Demarest et al. 2006: Fig. 7).

captura de Chak ... B'ahlam. De ser así, el dominio de Cancuén habría sido entonces de sólo cuatro años de duración. Pero existen, sin embargo, otras dos menciones a Machaquilá en Cancuén anteriores al 795 d.C. Una está en la Estela 1 de Cancuén, dedicada hacia el 790 d.C. En ella se menciona el Glifo Emblema de Machaquilá, aunque el contexto, desgraciadamente, no está claro en las reproducciones existentes del monumento. La otra mención aparece en la Escalera Jeroglífica 1 de Cancuén, un ritual de fuego que pudo realizarse en Machaquilá, quizá asociado a la fecha de 9.17.15.4.14 8 Hix 13 Pop, el 786 d.C. (Fahsen et al. 2003: 708-710, y Fig. 11), cuatro años antes de la fecha de la Estela 1. Si estas menciones a Machaquilá implican ya una subordinación a Cancuén, éste se produjo entonces al menos desde el 786 d.C., sugiriendo un periodo de dominio de 13 años. Como mucho, el periodo de dominio se extendió desde el año 776 (última fecha de la Estela 18 de Machaquilá) al 799 d.C., es decir, no más de veintitrés años. La unión de las dos coronas supuso una concentración de recursos económicos en Cancuén, en términos de tributación y posiblemente afluencia de mano de obra, coincidiendo con las últimas grandes obras de construcción de su palacio real y el crecimiento urbano de la ciudad.

De esta época de unión de las coronas en la persona del rey de Cancuén procede un interesante monumento, el Panel 3 de Cancuén, un panel exquisitamente labrado, el cual fue hallado durante las excavaciones en el palacio de Cancuén (Figura 8) (Demarest et al. 2006). En el monumento, el rey Tajal Chan Ahk aparece sentado sobre un pedestal teratomorfo, llevando el tocado de la Serpiente de Agua, flanqueado por dos cortesanos arrodillados. La escena se enmarca en un espacio cuadrilobulado, similar al recinto ritual construido que se encuentra en la Plaza A de Machaquilá. El texto jeroglífico se refiere expresamente a ese espacio cuadrilobulado, el cual se menciona como «la casa de Tajal Chan Ahk», donde se celebra el ritual calendárico de conmemoración del primer ho tuun en 9.18.5.0.0.0 (795 d.C.). Federico Fahsen correctamente ha relacionado este recinto cuadrilobulado con el de la Plaza A de Machaquilá, indicando que el ritual de conmemoración tuvo lugar en esta última ciudad. Los personajes arrodillados que flanquean a Tajal Chan Ahkul de Cancuén en el panel están identificados en el texto como un ajk'uhu'n o sacerdote, a la derecha, y como 
un noble de rango sajal, a la izquierda. Es la mención al sajal lo que nos interesa más en este caso, porque puede estar indicándonos que, durante este periodo, Machaquilá fue gobernado desde Cancuén mediante un noble de la corte de Cancuén destacado como gobernador provincial, una de las funciones que se han señalado para el cargo sajal (Houston 1993; Houston y Stuart 2001; Houston e Inomata 2009).

\subsection{La restauración de la soberanía de Machaquilá y el fin de la dinastía $(800->841$ d.C.)}

El dominio de Cancuén sobre Machaquilá tocó a su fin el año 800 d.C., cuando subió al trono de Machaquilá Ochk'in Kalo'mte', separando de nuevo ambas coronas. El año y medio que media entre la última fecha conocida de Tajal Chan Ahk en el Panel 3 de Cancuén realizando un ritual en la tumba de su predecesor, en enero de 799 d.C., y la fecha de accesión al trono de Ochk'in Kalo'mte, en junio de 800 d.C., fueron sin duda cruciales para Machaquilá. En ese periodo se produce la muerte del poderoso Tajal Chan Ahk y, con su sucesión en Cancuén, el surgimiento de una oportunidad a las aspiraciones de Ochk'in Kalo'mte', quien se sintió en ese momento con suficiente fuerza y apoyos para reclamar el título de rey de Machaquilá. Aunque K'an Maax, el efímero sucesor de Tajal Chan Ahk en Cancuén, ostenta en una ocasión el doble Glifo Emblema, los acontecimientos se desarrollaron finalmente a favor del candidato de Machaquilá. Quizá formando parte de la misma sucesión de eventos, Cancuén será destruido en o poco después de 800 d.C., falleciendo K'an Maax, el último monarca conocido de Cancuén. Tras el ascenso al poder de Ochh'ki'n Kalo'mte' de Machaquilá y la muerte de K'an Maax de Cancuén, el Glifo emblema de Machaquilá sólo volverá a ser llevado por los gobernantes de este último sitio.

Ochk'in Kalo'mte' continuó el programa de sus predecesores en Machaquilá, erigiendo dos estelas en la Plaza A, las estelas 1 y 2 . Es de la larga inscripción de la Estela 2 (Figura 9) de donde procede la información epigráfica que tenemos para su reinado 9 . Nacido en $916.19 .10 .19,3$ de septiembre de 770 d.C., accedió al poder en 9.18.9.15.10 12 Ok $18 \mathrm{Mol}, 28$ de junio de 800, a la edad de veintinueve años.

No hay constancia explícita de su filiación de parentesco, pero como su nacimiento se produce algo más de cinco años antes de la dedicación de la Estela 18, es entonces posiblemente hijo del gobernante que dedica dicho monumento, que aquí hemos considerado que es Chak ... B'ahlam, el monarca de Machaquilá capturado por las tropas de Cancuén. Aunque ya hemos comentado que la fecha de la derrota y captura de Chak ... B'ahlam no es conocida, quizá si se produjo cuando Och K'in Kalo'mte' era aún un niño o un adolescente, su corta edad en ese momento sin duda contribuyó a que sus derechos al trono quedaran relegados en favor de las pretensiones de Tajal Chan Ahk, entonces en la cúspide de su poder político y militar.

\footnotetext{
${ }_{9}^{9}$ Aunque el texto de un costado de la estela menciona el Fin de Periodo 9.19.0.0.0 8 Ajaw 18 Mol, 810 d.C., y es la fecha que se suele considerar como la de dedicación del monumento, en realidad se menciona en el contexto de la referencia al Medio Periodo anterior de 9.18.10.0.0. Del reinado de Ochk'in Kalo'mte' sólo conocemos eventos contemporáneos asociados al periodo 800-801 d.C., recogidos en la Estela 2. La propia estela se dedica en 9.18.10.7.5 12 Chikchan 13 Kumk'uh, el 9 de enero de 801 d.C. No disponemos de información acerca de los otros catorce años que presumiblemente duró su gobierno.
} 


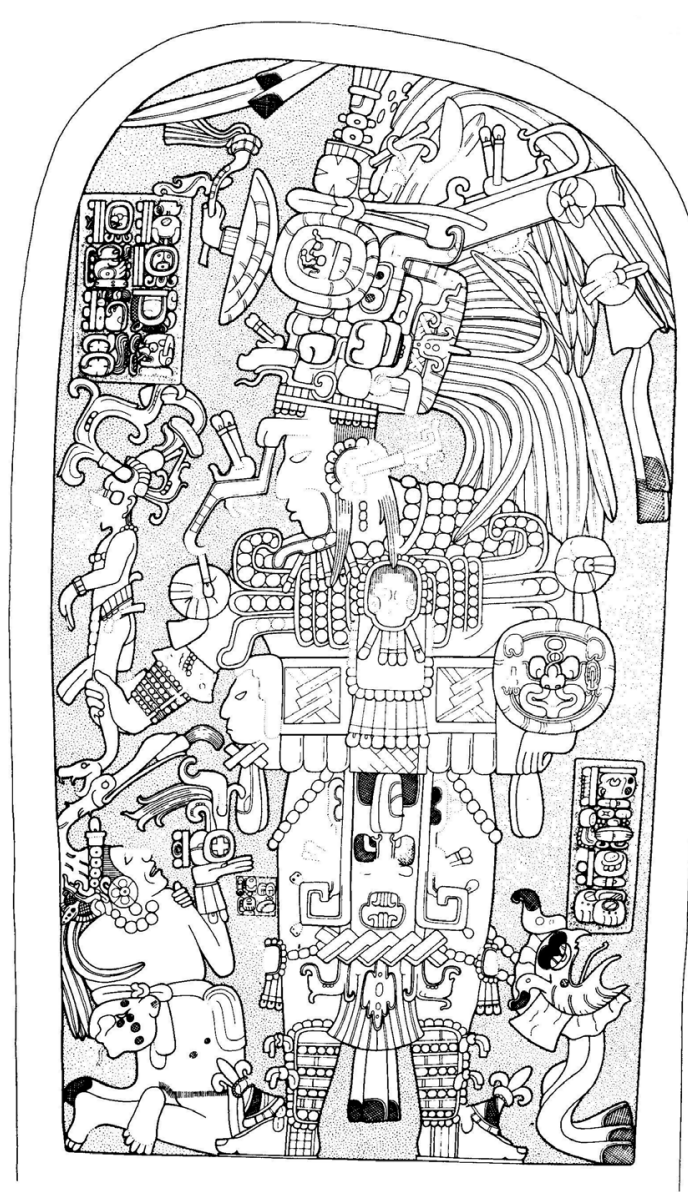

Figura 9: Machaquilá, Estela 2 (según dibujo de I. Graham [1967: Fig. 44]).
En la Estela 2, Ochk'in Kalo'mte' aparece representado armado de escudo y exhibiendo el cetro real del dios K'awiil, vestido como el dios de la lluvia. Ostenta los altos títulos de k'uhul 174-su ajaw, «rey sagrado de Machaquilá», y b'aah kab' "príncipe de la tierra». El nombre elegido por el gobernante en su accesión al trono, Ochk'in Kalo'mte', «el Kalo'mte' del oeste», no fue casual. Ochk' in Kalo'mte' es en realidad, en origen, el título que llevaba Siyaj K'ahk', el personaje asociado a Teotihuacan que, cuatrocientos veintidós años antes, en 378 d.C., protagonizó el evento de «llegada» a Tikal. La relación explícita se hace entre el nombre del rey y ese episodio (al que también está vinculada la erección de las estelas de Tres Islas en Clásico Temprano con iconografía teotihuacana que antes vimos) constituye el fundamento de legitimación con que se arropa el gobernante de Machaquilá en su entronización, una referencia a los gloriosos tiempos pasados en los que Machaquilá contaba con aliados poderosos, todo un programa político: Ochk'in Kalo'mte' no es

sólo un gobernante más de la secuencia dinástica de Machaquilá; es un verdadero restaurador. Esta vinculación a Teotihuacan de Ochk'in Kalo'mte' a través de su nombre pudo ser también una réplica consciente a la propia vinculación que había hecho diez años antes Tajal Chan Ahk, cuando se retrató en la Estela 2 de Cancuén, hacia el 790 d.C. ${ }^{10}$, sentado en un trono con yelmo, escudo y dardos a modo de un guerrero teotihuacano, con un cautivo yaciendo a sus pies. Otro de los títulos ostentados por el gobernante en la Estela 2, ajho'b 'aak «el de los cinco cautivos», un título militar, sugiere una exitosa actividad guerrera, quizá el resultado de una serie de campañas militares necesarias para obtener de nuevo y afianzar la independencia del reino.

${ }^{10}$ Proskouriakoff (1950: 186) fecha las estelas 1 y 2 de Cancuén en 9.18.10.0.0? (800 d.C.) y 9.18.0.0.0 (790 d.C.); Fahsen y Demarest (2001: 862) las fechan en 790 d.C. y 800 d.C. En cualquier caso, por estilo, ambas estelas corresponden al final del siglo VIII. 
La Estela 2 también habla de la continuación de la actividad ceremonial llevada a cabo por el gobernante, con la celebración de rituales calendáricos en la Plaza A. Es posiblemente durante el reinado de Ochk'in kalo'mte' o su sucesor cuando se hace el diseño y remodelación final del recinto cuadrilobulado rehundido que hoy conocemos, presumiblemente en el mismo lugar en el que existió el anterior, al que sustituyó completamente.

La restauración de la soberanía de Machaquilá en la persona de Ochk'in Kalo'mte y su reinado de posiblemente quince años van a coincidir con dos acontecimientos políticos cruciales en la región, fechados por los arqueólogos a comienzos del siglo IX: la desaparición violenta de los reinos de Cancuén y de Aguateca (esta última, nueva capital de la dinastía real de Dos Pilas durante medio siglo), los dos enemigos tradicionales de Machaquilá. Ambos acontecimientos van a tener características similares: un asalto a la capital -completamente fortificada en el caso de Aguateca y en proceso de fortificación en el caso de Cancuén, cuyos habitantes no tuvieron tiempo de concluir sus defensas- y su posterior destrucción (para Aguateca, vid. Inomata 1997, Inomata y Triadan 2010). La extrema e inusual violencia del ataque es patente en Cancuén, donde recientemente se han hallado los restos masacrados de no menos de treinta individuos (hombres, mujeres y niños), posiblemente los miembros de la familia real (Barrientos et al. 2006; Demarest et al. 2006; Barrientos y Demarest 2007). Los cuerpos presentan evidencias de muerte violenta, y junto a los restos han sido recuperados numerosas puntas de proyectil. Ni siquiera hubo saqueo. Los cuerpos conservaban sus ricos adornos cuando, tras la matanza, fueron arrojados en una cisterna, frente al gran palacio construido por Tajal Chan Ahk. La tumba del último gobernante de Cancuén, K'an Maax, que sucedió brevemente a su padre, se halló a cierta distancia, sin gran pompa, con el cuerpo directamente enterrado en el barro del suelo.

Aunque tanto los arqueólogos de Aguateca como los de Cancuén han señalado a Machaquilá como posible responsable de la destrucción de sus respectivas ciudades, lo cierto es que no hay de momento ninguna mención epigráfica explícita en Machaquilá que permita afirmar o negar esta autoría. Motivos de revancha no hubieran faltado, sin duda: la agresividad de la dinastía intrusiva de Dos Pilas-Aguateca inaugurada por B'ajlaj Chan K'awiil en el río Pasión fue posiblemente la causante del traslado de la capital de Machaquilá a su nuevo emplazamiento a finales del siglo VII, como antes se sugirió, y el recuerdo del dominio de Cancuén sobre Machaquilá tras la derrota y muerte de Chak ... B'ahlam estaba aún fresco en la memoria -hemos especulado con la posibilidad de que Ochk'in Kalo'mte' fuera su hijo-. Es posible que Ochk'in Kalo'mte', ya un adulto de casi treinta años, utilizara el recurso de la guerra para hacer valer sus derechos dinásticos al trono tras la muerte de Tajal Chan Ahk. Es cierto que, dado que los ataques a Cancuén y Aguateca se fechan arqueológicamente hacia el 800 (Demarest et al. 2006) y el 810 d.C. (Inomata et al. 2004; Inomata y Triadan 2010: 11), Ochk'in Kalo'mte' pudo haber sido el artífice. En cualquier caso, con independencia del papel que jugara Ochk'in Kalo'mte' en estos acontecimientos, lo cierto es que Machaquilá se benefició largamente de la desaparición de los que eran los dos reinos contemporáneos más poderosos de la zona. Machaquilá inicia el siglo IX renovada y fortalecida, sobreviviendo a otras entidades políticas del área durante medio siglo más. 


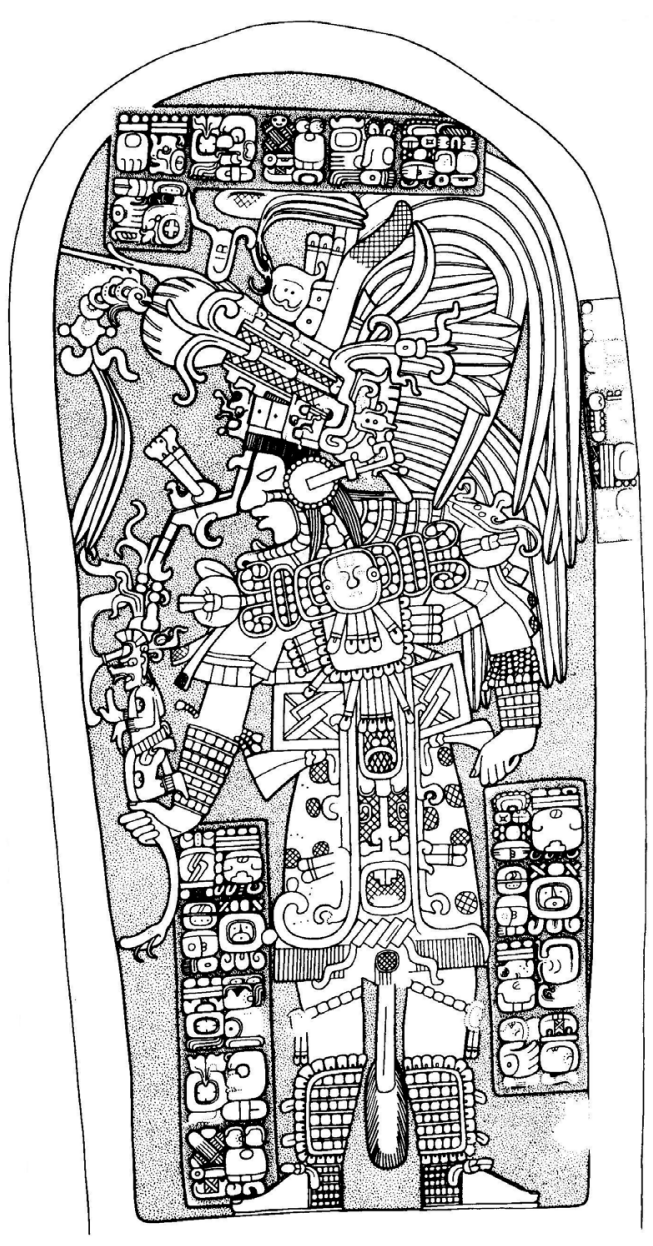

Figura 10: Machaquilá, Estela 3 (según dibujo de I. Graham [1967: Fig. 49]).

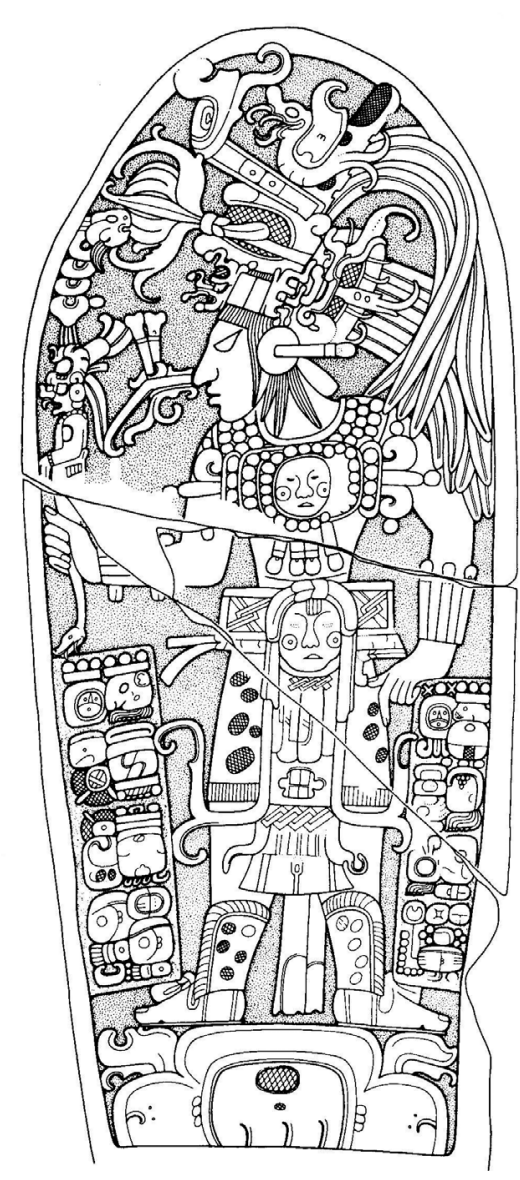

Figura 11: Machaquilá, Estela 4 (según dibujo de I. Graham [1967: Fig. 51]).

El sucesor de Ochk'in Kalo'mte', el rey Siyaj K'in Chaahk II, subió al trono en 815 d.C. Nuevamente la elección de su nombre no parece casual: su homónimo Siyaj K'in Chaahk I fue posiblemente el autor del traslado de la capital del reino de su antiguo emplazamiento de Clásico Temprano a su ubicación actual. Siyaj K'in Chaahk II está asociado a las fechas de $815,816,820$ y 821 d.C., que aparecen mencionadas en las estelas 3 y 4 que erige en la Plaza A, junto a las de su predecesor, retratándose como él con el cetro real del dios K'awiil (Figuras 10 y 11). Como su predecesor también, Siyaj K'in Chaahk II continúa con el discurso político legitimador vinculado a Teotihuacan (Just 2006: 98-99). Además del título de k'uhul 174-su ajaw «rey sagrado de Machaquilá», exhibe el título T600-TE'-NAH pi-tzi-li, [Wi]te'naah pitzil «jugador de pelota(?) de Wite'naah», el mismo topónimo wi-TE'-NAH, Wite'naah vinculado con Teotihuacan mencionado en la Estela 2 de Tres Islas. En la Estela 3, después del 
título «rey sagrado de Machaquilá» del Glifo Emblema, añade el alto título kalo'mte'. El uso de este título por un rey de Machaquilá es ciertamente interesante, en cuanto a que, en toda la región del Pasión, sólo algunos reyes de Dos Pilas-Aguateca lo habían ostentado hasta su caída hacia el 810 d.C. Quizá Machaquilá sí tuvo que ver finalmente con la caída de la dinastía de Aguateca -y, por las fechas y el título, más que Ochk'in Kalo'mte', fue Siyaj K'in Chaahk II el autor del ataque-. En cualquier caso, se consideró su heredera.

Resulta muy interesante que en la Estela 4 (vid. Figura 11) Siyaj K'in Chaahk II se retrate sobre el icono del espacio cuadrilobulado, el recinto ritual de la Plaza A. Entre el abundante material recuperado en las excavaciones del recinto cuadrilobulado, apareció un fragmento de un vaso inciso con los restos de la inscripción ..] pi-tzi [... (vid. Lacadena 2006: Fig. 18c). Dado que de todos los reyes conocidos de Machaquilá sólo Siyaj K' in Chaahk II ostenta el título de Wite'naah pitzil, es posible que los dos signos conservados en el fragmento cerámico se refieran a este título -[Wite'naah] pitzi[l]-, lo que sitúa al rey actuando ritualmente en el recinto, como sugería la representación iconográfica, dejando como ofrenda ceremonial o como objeto del ritual un vaso con su nombre.

Del sucesor de Siyaj K'in Chaahk II no sabemos prácticamente nada. No se le conocen monumentos propios, posiblemente por la brevedad de su reinado, que debe situarse en los apenas tres años que median entre la última fecha conocida de Siyaj K'in Chaahk II de 821 d.C. y la primera fecha asociada a otro gobernante, Jun Tzak Tok', en 824 d.C ${ }^{11}$. Ni siquiera sabemos el nombre de este rey. Sólo el título guerrero de u-cha-CHAN-na \#-b'u-lu-K'AK', uchan ...b'ul K'ahk' «el guardián de ...b'ul $K^{\prime} a h k^{\prime} »$, con el que su hijo y sucesor se refiere a él en la Estela 6. Podemos afirmar que fue rey de Machaquilá por los títulos de «rey sagrado de Machaquilá» y «príncipe de la tierra» que le acompañan en la mención única que de él tenemos ${ }^{12}$.

La siguiente accesión al trono conocida en Machaquilá es la de Jun Tzak Tok', en 824 d.C. Su reinado fue relativamente largo, de al menos dieciséis años, con fechas asociadas de $824,825,830,831,835,836$ y 840 d.C. Jun Tzak Tok' erigió un total de cinco estelas, cuatro de ellas labradas, en la Plaza A, las estelas número 5-9, al pie de la Estructura 19, alineándolas a continuación de las estelas de Ochk'in Kalo'mte' y Siyaj K' in Chaahk II. En las estelas que comisiona, Jun Tzak Tok' se retrata eligiendo motivos iconográficos tradicionales de sus antecesores, como la representación del tocado de la Serpiente de Agua, la exhibición del cetro del dios K'awiil o la representación del monarca sobre el icono del recinto cuadrilobulado (estelas 7 y 8), que sigue siendo el lugar ceremonial más importante del sitio (Figura 12). Otros elementos, en cambio, son innovadores, como dos de los tocados con los que se representa, de ave (Estela 5) y de jaguar (Estela 6). Es en su reinado cuando la técnica escultórica de los

\footnotetext{
${ }^{11}$ En los tres presuntos años de duración de este reinado no se cumplió ninguna de las efemérides calendáricas típicamente conmemoradas en Machaquilá en esta época (finales de ho tuun de 5x360 días y el ciclo de $5 \times 365$ días asociado), por lo que quizá no le correspondió erigir ninguna estela en la Plaza A.

12 Just (2006: 139) no considera que este título se refiera a un nuevo gobernante situado entre Siyaj K'in Chaahk II y Jun Tzak Tok', sino que se refiere a alguno de los gobernantes precedentes, Ochk'in Kalo'mte' o Siyaj K'in Chaahk II. Hasta que aparezca nueva información, ésta es una posibilidad, evidentemente, a tener en cuenta.
} 


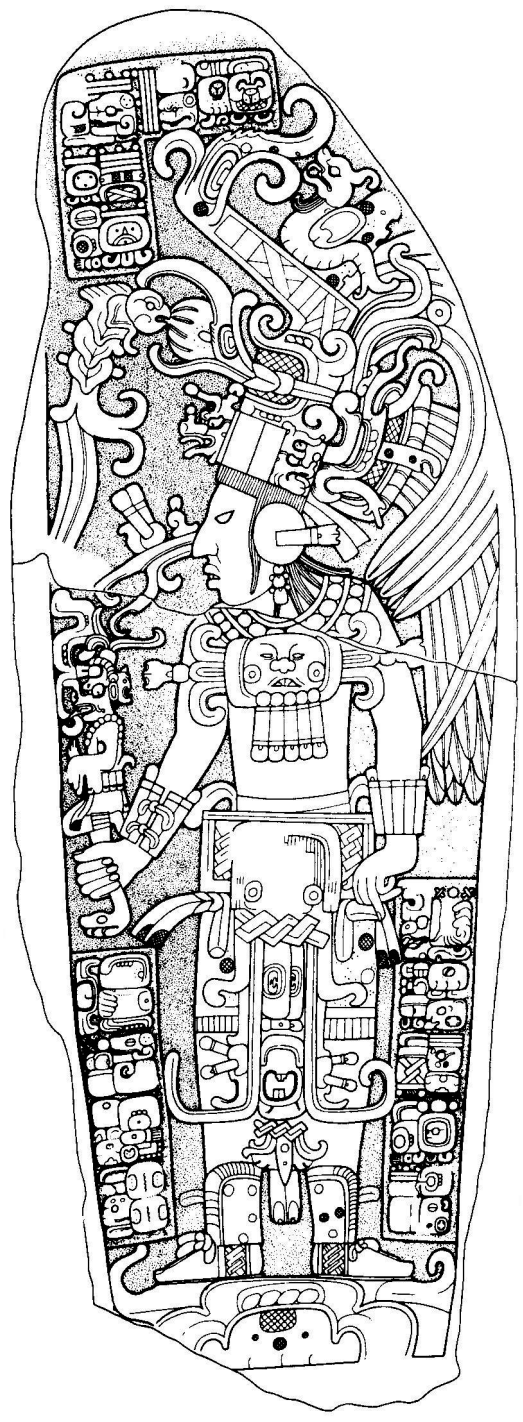

Figura 12: Machaquilá, Estela 7 (según dibujo de I. Graham [1967: Fig. 57]). artistas de Machaquilá culmina un proceso de madurez adquiriendo una fuerte personalidad propia (vid. Just 2006: 107-148).

Jun Tzak Tok' se asocia a los títulos de k'uhul 174-su ajaw «rey sagrado de Machaquilá» y b'aah kab' "príncipe de la tierra». Al menos en tres de los monumentos exhibe el título guerrrero u-cha-CHAN-na IK'-b'o-b'o TOK', uchan Ik'B 'ob'Tok', «el guardián de Ik' B' ob' Tok' ${ }^{13}$ lo que le señala como gobernante exitoso en asuntos militares. Conservamos su filiación, registrada en la Estela 6, en la que se declara hijo de una princesa real de un reino desconocido (posiblemente de la región) y del rey mencionado con el título uchan ...b'ul K'ahk' "el guardián de ...b'ul k'ahk'». La erección de monumentos y el cumplimiento de los rituales calendáricos son el tema central de los textos de sus estelas. La Estela 5, de 10.0.10.17.5 13 Chikchan 13 Kumk'uh, 840 d.C., es el último monumento fechado erigido en la Plaza A.

El nombre de un nuevo gobernante del siglo IX en Machaquilá fue identificado en el transcurso de las excavaciones del proyecto hispano-guatemalteco, con la recuperación de nuevos fragmentos glíficos de la Estructura 4 de la Plaza F del sitio, el llamado «Escorpión» Ti' Chaahk (Iglesias y Lacadena 2003; Ciudad et al. 2004; Lacadena e Iglesias 2005). Aunque no se ha conservado ninguna fecha absoluta en las inscripciones asociadas a su reinado, ciertos indicios arqueológicos y epigráficos aconsejan ubicarlo en el siglo IX. Hasta contar con nueva evidencia, hemos sugerido ubicarlo entre los reinados de Ochk'in Kalo'mte' y Siyaj K’in Chaahk II, o bien -quizá más probable esta última opción-, inmediatamente a continuación de Jun Tzak Tok', reinando, por

\footnotetext{
${ }^{13}$ Este cautivo suele ser designado en la literatura como B'ob' Tok'. En las estelas 5 (B3) y 6 (A5) de Machaquilá los signos b'o del nombre B'ob' Tok' presentan achurado en su superficie interior, lo que es ciertamente peculiar, dado que el achurado no forma parte del diseño del fonograma b'o. En Ek' B'alam, un gobernante es nombrado como K'an B'ohb' Tok' o K'an B'ob' Tok' (vid. Lacadena 2003), con el color k'an «amarillo» precediendo la secuencia B'ohb'/B'ob' Tok'. Es posible que el achurado indique en Machaquilá el color IK', $i k$ ' «negro», y que el nombre completo del cautivo sea, por tanto, Ik' B'ob' Tok', lectura que sigo aquí.
} 


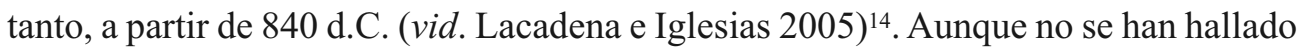
estelas erigidas durante su reinado, conocemos a «Escorpión» Ti' Chaahk por dos menciones en los fragmentos de sillares glíficos recuperados en la Estructura 4 de la Plaza F (Iglesias y Lacadena 2003). Estos sillares y fragmentos de sillares contienen los restos de lo que fue una larga inscripción ${ }^{15}$. Aunque algunos investigadores se han referido a ella como una escalera o incluso un friso jeroglífico, la forma y distribución de los fragmentos apuntan a que pudieron formar originalmente una banqueta o trono de mampostería que se ubicó en el interior del cuarto principal de la estructura, en su última remodelación (Figura 13a). El formato sería parecido al de la Banca Jeroglífica 1 de Dos Pilas (Lacadena e Iglesias 2005) (Figura 13b).

Aunque la inscripción en su estado actual se encuentra aún incompleta y desordenada -en la imagen de la Figura 11a sólo los tres primeros bloques glíficos de la izquierda conteniendo los restos de una fecha y los cuatro sillares cóncavos de la última de las presumibles patas con el nombre y títulos de «Escorpión» Ti' Chaahk se encuentran posiblemente en la posición original-, podemos saber algo de su contenido. La inscripción conmemora el aniversario de un $k$ 'atun (veinte años) de un evento, y la llegada de un personaje a Machaquilá (i-HUL-li, $i$ huli «entonces llegó»). Este personaje es quizá una princesa de sangre real del vecino reino de Mahk ${ }^{16}$, quien podría ser la esposa del rey «Escorpión» Ti’ Chaahk. El lugar al que llega la princesa de Mahk se nombra como «la Casa de la Banda (Real) de Palacio»-posiblemente el nombre del complejo palaciego al que pertenece la Estructura 4-17. La localización del reino de Mahk no es conocida, debiendo ubicarse probablemente en la vecindad de Machaquilá (Zender 2005). Además del rey y la reina, la inscripción menciona otros personajes, al menos dos femeninos (uno de los cuales lleva el gentilicio de Machaquilá, y ella misma o la otra se asocian al término de parentesco ya-na-MURCIÉLAGO «la madre de») y uno masculino (vid. Lacadena e Iglesias 2005).

«Escorpión» Ti' Chaahk lleva los títulos de k’uhul 174-su ajaw «rey sagrado de Machaquilá» y "veintiocho» b'aah kab' "príncipe de la Tierra», como los otros gobernantes del siglo IX Ochk'in Kalo'mte' y Jun Tzak Tok'. No tenemos información sobre su actividad ritual en la Plaza A.

Tras las menciones a «Escorpión» Ti' Chaahk, a mediados del siglo IX se produce el silencio epigráfico de Machaquilá. Si bien hay evidencia de ocupación y construcción en Clásico Terminal en el sitio, algunos edificios quedan inconclusos y quizá hay

\footnotetext{
${ }^{14}$ Just (2006: 60, 67), sin embargo, sugiere ubicar a este nuevo gobernante entre «Etz'nab'» Chaahk, cuya última fecha conocida es de 761 d.C., y el gobernante que dedica la Estela 18 en 776 d.C.

${ }^{15} \mathrm{Me}$ refiero a los fragmentos de mayor tamaño del conjunto y a los inscritos en medallones. Los fragmentos de formato menor pertenecen a una inscripción independiente.

16 Tradicionalmente se ha leído el nombre del reino de la princesa como Ahk, poniéndose en relación con el topónimo Ahkul mencionado en Dos Pilas (Houston 1993: 118, fig. 4.22a; Lacadena e Iglesias 2005: 658). Zender (2005) ha ofrecido recientemente clara evidencia de que el logograma con el que se escribe el nombre del reino no es $\mathbf{A}(\mathbf{H}) \mathbf{K}$ sino $\mathbf{M A}(\mathbf{H}) \mathbf{K}$.

${ }^{17}$ Marc Zender (2005: Nota 10) coincide en que los bloques glíficos que preceden el título de la princesa de Mahk podrían referirse al nombre de la estructura arquitectónica. Tras una nueva inspección del sillar que contiene la referencia, Zender ( ibid.) propone que el primer signo erosionado puede leerse como una variante aviana del logograma CHAN «cielo», en vez de HUN «banda», y que el logograma OTOT contiene infijo el logograma TUN, que debe incorporarse a la lectura. Según este autor, entonces, el nombre de la estructura sería Chanal Naaj Tuun Otoot «Heavenly House (of the) Stone Dwelling».
} 

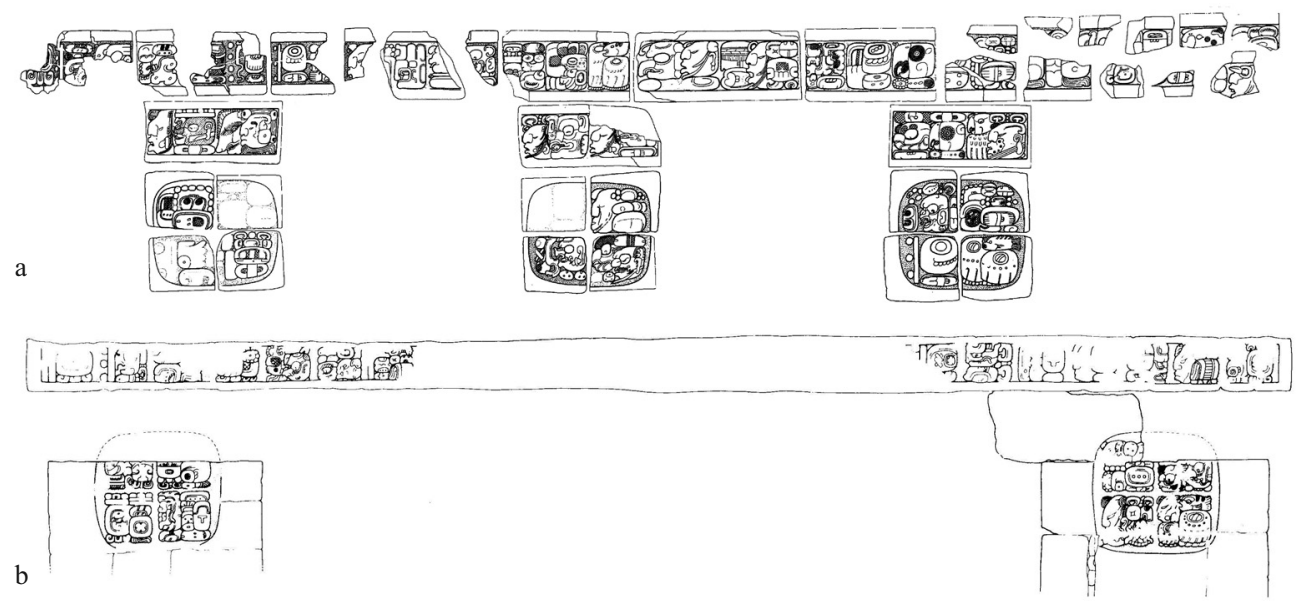

Figura 13: Los fragmentos jeroglíficos de la Estructura 4: a= Reconstrucción de la presunta Banca Jeroglífica de la Estructura 4 (según composición de A. Lacadena, basada en dibujos de I. Graham y A. Lacadena); b= Banca Jeroglífica 1 de Dos Pilas (según dibujo de S. Houston [1993: Fig. 4-9]).

evidencia de destrucción de la propia banca jeroglífica de la Estructura 4 que menciona a «Escorpión» Ti' Chaahk, la cual es desmantelada ya en época Clásica. El desmantelamiento y destrucción de tronos reales, como símbolos de autoridad, aparecen en contextos de derrota militar-como la destrucción del Trono 1 de Piedras Negras (Martin y Grube 2000: 153)-. Los rituales dinásticos de la Plaza A se suspenden y no hay evidencia de más erecciones de estelas y monumentos públicos. El estudio de la cerámica hallada en el recinto ritual cuadrilobulado fecha su construcción y uso en la transición de Clásico Tardío a Clásico Terminal, entre 800-850 o entre 830-880 d.C. (Lacadena 2006: 110-111), lo que concuerda bien con las fuentes epigráficas.

No podemos dejar de relacionar el silencio de Machaquilá con la restauración del poder real en Seibal, ubicada sobre el río Pasión al noroeste de Machaquilá, cuya dinastía comienza a erigir monumento con inscripciones en 849 d.C. (Schele y Grube 1995; Schele y Mathews 1998; Just 2006: 147-148). Resulta significativo que el nuevo gobernante de Seibal, wa-t'u-lu-k'a-te-le, sea quien ostente ahora el título kalo 'mte'. En el nuevo orden político del Clásico Terminal en la región, la dinastía de Machaquilá no tuvo ya cabida.

\section{Rituales dinásticos en la Plaza A de Machaquilá}

Uno de los lugares más interesantes en Machaquilá es el pequeño espacio de la Plaza A donde se concentra la mayor parte de las estelas del sitio y, dentro de ese espacio, el recinto rehundido de forma cuadrilobulada. Su interés proviene del hecho de que aunque el cuadrilobulado es un motivo ubicuo en representaciones iconográficas no solo mayas sino también mesoamericanas, como elemento físico construido es excepcional. Recintos similares han sido hallados en La Blanca (Love et al. 2006) 
y Aguacatal (Houston et al. 2005), datados en Preclásico Tardío y Clásico Temprano. De ellos, el de Machaquilá, con sus aproximadamente 8,40 x 8,40 m, es de momento el de mayores dimensiones. Este recinto fue descubierto por Graham (1967: 59, y Fig. 42), quien lo incluyó como parte del dibujo de detalle de la planta de la Plaza A con la distribución de las estelas, si bien no pudo entonces presentar su contorno completo. Se debe a Stuart y Houston (1994: 33, y figs. 37 y 38) la identificación de este singular recinto construido en la Plaza A con los iconos trilobulados y cuadrilobulados representados a los pies de los monarcas en algunas de las estelas del sitio. En el contexto de los trabajos arqueológicos del equipo hispano-guatemalteco, se abordó en la temporada de campo de 2005 la excavación del mismo, con objeto de conocer definitivamente su forma y tratar de determinar los rituales llevados a cabo en dicho espacio y su relación con la iconografía y las inscripciones jeroglíficas de los monumentos asociados de la Plaza A. Tras las intervenciones arqueológicas, se pudieron determinar la forma y dimensiones del mismo -confirmando que se trataba de un recinto cuadrilobulado completo-, terminando el dibujo en planta que Graham no pudo concluir y recuperando gran cantidad de material arqueológico (Chocón et al. 2007; Lacadena 2006; Ciudad et al. 2010) (vid. Ciudad et al., en este volumen: Figs. 3 y 4).

A la hora de comprender qué tipo de rituales se realizaban en asociación con el recinto cuadrilobulado es preciso tener en cuenta en primer lugar su ubicación. El recinto se encuentra en el fondo este de la Plaza A, una plaza de mediano tamaño, con el acceso principal desde la Plaza C por el oeste, y dando paso, a su vez, a la Plaza B por el sur (vid. Figura 1). La Plaza A no forma parte de las grandes zonas públicas de Machaquilá concebidas para la concentración de grandes contingentes de población, como son la Plaza C y la Plaza D. Este hecho nos ofrece ya un primer rasgo importante de los rituales asociados con recinto cuadrilobulado: unos rituales realizados en un espacio de acceso restringido, no destinados a ser vistos por grupos grandes de asistentes pertenecientes a un abanico amplio de la sociedad, sino a un grupo más exclusivo, posiblemente todos ellos pertenecientes a la élite política, social y religiosa del reino (Ciudad et al. 2010: 141-145; Ciudad y Adánez, en este volumen). Esta cualidad de acceso restringido posiblemente se acentuó con el tiempo, incrementándose en la primera mitad del siglo IX, cuando la Plaza A adopta su forma definitiva.

Aunque arqueológicamente el recinto cuadrilobulado que ahora conocemos se construyó y estuvo en uso en las primeras décadas del siglo IX (Lacadena 2006: 110-111), es muy posible que existiera una versión anterior del recinto. La iconografía muestra a gobernantes de Machaquilá asociados al icono del cuadrilobulado no sólo en las estelas del siglo IX -como en las estelas 4 (de Siyaj k'in Chaahk II, en 821 d.C.), y 8 y 7 (de Jun Tzak Tok', en 825 y 831 d.C.)-, sino también en las del siglo VIII -como se aprecia en las estelas 10 y quizá 12 (de «Etz'nab'» Chaahk, la segunda de 761 d.C.), y 18 y 19 (de Chak ... B'ahlam?, la primera de 776 d.C.) (vid. Figuras 6, 11 y 12)-. Esto nos habla de la continuidad y mantenimiento de un espacio de profunda significación simbólica, integrado no sólo por un recinto en el suelo de la plaza y unos monumentos que se disponen en su cercanía, sino también por todo un complejo arquitectónico compuesto por las estructuras piramidales que cierran los costados norte y este de la plaza y que constituyen su marco de fondo. El espacio ritual de la Plaza A se fue construyendo paulatinamente por los gobernantes de la dinastía. 
Los rituales llevados a cabo en el cuadrilobulado están relacionados con el agua y las deidades asociadas al agua ${ }^{18}$. Esta asociación está indicada, en primer lugar, por el icono de agua que en las estelas de Machaquilá suele acompañar el motivo cuadrilobulado de las bases de las estelas que lo representan (como se aprecia en las figuras 6 y 12) ${ }^{19}$; en segundo lugar, por los seres sobrenaturales vinculados con el agua que lo habitan: uno es el propio dios de la lluvia, Chaahk, que emerge del espacio cuadrilobulado en la base de la Estela 10 (Houston et al. 2005: 5-7; Just 2006: Cap. 2, p. 46; García Barrios 2009: 244-249) (vid. Figura 6); otro es la Serpiente de Agua, a quien personifican los gobernantes de Machaquilá en sus tocados (para una detallada descripción iconográfica, vid. Just 2006: Cap. 2) ${ }^{20}$; y un tercero es la gran tortuga que nada en el agua primordial y en cuyo caparazón suele abrirse un espacio cuadrilobulado, ser representado en el gran Altar A situado a poca distancia al oeste del recinto cuadrilobulado (vid. Graham 1967: Figs. 71 y 73) ${ }^{21}$.

Todos estos elementos asociados al recinto cuadrilobulado de Machaquilá aparecen ya representados juntos en los Murales de San Bartolo, hacia el siglo I a.C., donde en una de las escenas del Muro Oeste se muestra a la tortuga cósmica con el cuadrilobulado abierto en su caparazón, en cuyo interior se encuentran sentados Chaahk y la Serpiente de Agua en forma antropomorfa flanqueando al dios del maíz, el cual baila con un tambor a la espalda golpeando un caparazón de tortuga con astas de venado (Saturno et al. 2005). La indicación de música y baile en esta temprana representación de San Bartolo es sumamente interesante, en cuanto a que en Machaquilá, en dos monumentos del siglo VIII, las estelas 10 y 17, los gobernantes muestran un talón levantado sugiriendo que están bailando (Houston et al. 2005; Just 2006: Cap. 2, passim). En el caso de la Estela 10, este baile se asocia claramente con el recinto cuadrilobulado.

En San Bartolo, en la escena de la tortuga con el cuadrilobulado, las deidades del agua y el dios del maíz se encuentran al lado de una de las primeras representaciones de un rito de accesión de un gobernante maya, quien se encuentra sentado sobre una estructura de madera en el momento de recibir los atributos del cargo (Saturno et al. 2005). Esta asociación es particularmente interesante para Machaquilá, pues muestra la especial relación de este recinto y su simbología con la dinastía local y con la propia sucesión de los gobernantes (ritos de accesión se mencionan al menos en tres de los monumentos esculpidos de la plaza; asimismo, también en la misma plaza, son seis las representaciones de gobernantes que se muestran portando el cetro del dios K'awiil); y posiblemente también con la conjuración de los ancestros reales, vinculados

\footnotetext{
${ }^{18}$ Sin embargo, aunque la relación del recinto cuadrilobulado con el mundo acuático y deidades y seres sobrenaturales del agua es clara, aparentemente el recinto cuadrilobulado de Machaquilá no fue diseñado para contener agua. La Plaza A en la que se encuentra el recinto cuadrilobulado de Machaquilá estaba construida con una ligera pendiente, cuyo objetivo era posiblemente ayudar a la evacuación del agua más que a retenerla (Lacadena 2006: 109).

${ }^{19}$ Otros cuadrilobulados -ya sean construidos o representados iconográficamente-, como el de Aguacatal (Houston et al. 2005) o el del Panel 3 de Cancuén, muestran bandas acuáticas o burbujas en su contorno.

${ }^{20}$ Estelas 2, 3, 4, 7, 8, 10, 18 y 19 -las dos últimas ubicadas en la Plaza C-, y el personaje representado en el interior de la tortuga del Altar A.

${ }^{21}$ La Columna 1 de Ek' Balam representa a este ser y lo nombra como SAK-a-ku, Sak A[h]ku['l] (vid. Lacadena 2003). En yucateco colonial, zac aac se traduce como «hicoteas o tortugas o galápagos de agua dulce» (Arzápalo 1995: 151).
} 
al renacimiento del dios del maíz surgiendo del caparazón de la tortuga cósmica (Ciudad et al. 2010).

Las excavaciones del recinto recuperaron una gran cantidad de restos materiales (platos, vasos, cuencos, jarras, ollas, comales, incensarios, pequeñas cajas de cerámica con restos de pintura azul, silbatos, figurillas, puntas de pedernal, navajas de obsidiana, y restos humanos), en unas proporciones muy elevadas: casi 10000 fragmentos cerámicos fueron recuperados en $25 \mathrm{~m}^{2}$ de superficie interior excavados, algo más de un tercio de de la superficie total). Estos objetos pudieron ser depositados como ofrendas o como restos de instrumentos utilizados durante el ceremonial. La cantidad de restos hallados sugiere que una vez que se realizaba un ritual los objetos no se retiraban sino que se dejaban en el interior del recinto (Lacadena 2006; Ciudad et al. 2010). El tipo de recipientes recuperados sugiere preparación y consumo u ofrenda de alimentos, reales o simbólicos.

Muchos fragmentos cerámicos estaban abrasados, lo que señala el fuego como un elemento sumamente importante de los rituales allí realizados (Lacadena 2006; Ciudad et al. 2010). Ya hemos mencionado antes el interesante pasaje en la Escalera Jeroglífica de Cancuén donde hay una posible referencia a un evento relacionado con el fuego ( $u k^{\prime} a h k^{\prime}$ ' «su fuego») y su realización en Machaquilá (uhti T174-su-ji «ocurrió en «Machaquilá»»)). Es posible que exista una relación entre este ritual involucrando fuego y el patrón peculiar de fechas que se recoge en los monumentos de Machaquilá.

Ya Graham (1967: 97-98)22 detectó la existencia de un patrón de fechas recurrentes en las estelas 2 a 8 del siglo IX, consistente en la celebración de periodos de cinco años solares, que él a su vez dividió en dos series (I y II) según el año considerado fuera de $364(5.1 .0,1820$ días=5x364) o 365 días $(5.1 .5,1825$ días= 5x365), respectivamente ${ }^{23}$. Según Graham, ambos ciclos se distinguirían en incidir en la recurrencia de fechas con 1 Ajaw en el tzolk' in (los de 5X364 días, que se relacionarían con fechas 3, 8, 13 y 18 Kumk'uh en el haab') o 13 Kumk'uh en el haab' (los de 5x365 días, que se relacionarían con días Chikchan, Ok, Men y Ajaw en el tzolk'in). Estos ciclos de cinco años corren en paralelo a los propios ho'tuun o periodos de cinco años de 360 días propios de la aritmética de la Cuenta Larga. En las expresiones típicas de los monumentos de Machaquilá, se menciona la Rueda Calendárica del ho 'tuun de la Cuenta Larga en curso, y la Rueda Calendárica del periodo de cinco años solares inmediatamente siguiente. Riese (1988a) demostró que ya en la Estela 18 de la segunda mitad del siglo VIII, asociada al ho 'tuun 9.17.5.0.0, se mencionaba este otro ciclo paralelo, que caía en la fecha 9.17.5.1.0, demostrando de este modo una mayor antigüedad en el sitio para estas efemérides calendáricas.

Si bien los detalles se nos escapan, se ha sugerido (Ciudad et al. 2010: 141) que existe una relación entre estos rituales de fuego asociados a estas fechas y los rituales de los ahtoc «los quemadores» mencionados en los libros de Chilam Balam, asociados a las fechas 4 Ok-4 Men-4 Ajaw-4 Chikchan. En Machaquilá, la celebración de este ciclo de 365 días cae precisamente en tres de los cuatro días de esta serie ${ }^{24}$. Se señalaba

22 Vid. también una discusión en Just 2006: Cap. 2.: passim).

${ }^{23} \mathrm{El}$ ciclo es en realidad de 5.1 .5 o $5 \times 365$ días. Las presuntas anomalías incorporando otro ciclo de $5.1 .0 \mathrm{o}$ $5 \times 364$ días son sólo aparentes.

${ }^{24}$ Las fechas del ciclo registradas en Machaquilá (destacando en negrita los días del tzolk’in) son 9.17.5.1.0 
también que, interesantemente, pese a la clara relación con el fuego, existía una vinculación también estrecha entre estos rituales de los ahtoc y el dios de la lluvia, quien aparece visiblemente mencionado en los textos jeroglíficos y representado en las escenas asociadas del almanaque de las páginas $42 \mathrm{~b}-46 \mathrm{~b}$ del Códice de Dresde que recoge el ciclo de los quemadores.

Los textos jeroglíficos de Machaquilá también mencionan una actividad relacionada con los complejos rituales desarrollados en la Plaza A, la cual implica el cierre de una cámara. Esta actividad se menciona en tres ocasiones en los textos del sitio, como uma-ka-wa u-WAY-ya, umaka['] w uway «cerró su cámara» (Estela 2, K3-K4), u-maka-OL u-WAY-ya, umako'l uway «(ocurrió) el cierre de su cámara» (Estela 5, B3-B4) 25 , y u-b'a-la-wa u-WAY-ya-la, ub 'ala [']w uwayal «cubrió su cámara» (Estela 7, C1) (Lacadena 2006; Ciudad el al. 2010: 136 y Fig. 9). El término WAY-ya, way posiblemente se refiere a una cámara o cuarto ${ }^{26}$, y no al propio recinto cuadrilobulado, que tiene otro nombre en los textos jeroglíficos, escrito como T510-na ${ }^{27}$. La presunta cámara se encontraba en el interior del recinto rehundido, aunque fue destruida durante el saqueo que sufrió el altar o tapa que la cubría (vid. Lacadena 2006: 109-110).

Una última mención en los textos jeroglíficos de los monumentos erigidos junto al cuadrilobulado a las posibles actividades allí realizadas involucra al verbo $i l$ «ver», mencionado en dos de los textos (las estelas 3 y 7 , dedicadas por dos gobernantes distintos). Las fechas asociadas a estas dos menciones, 9.19.5.11.0 1 Ajaw 13 Kumk'uh en la Estela 3 y 10.0.0.14.15 3 Men 13 Kumk'uh en la Estela 7, pertenecen a la conmemoración del ciclo de $5 \times 365$ días. El verbo $i l$ «ver» suele formar parte de pasajes en los que se menciona a los asistentes o testigos de un evento mencionado previamente (yila «lo vio», ilaj «fue visto», yilaaj «lo ha visto»). El uso en Machaquilá de este verbo es, sin embargo, distinto, ya que no introduce el nombre de los testigos. En la Estela 3 (G3-G4) la expresión completa dice i-u-ti 1-AJAW 13-HUL-OL IL-ja K'AL-TUN-ni NAH-HO'-TUN-ni, i u[h]ti 1 Ajaw 13 Hul O'hl il[a]j k'altuun naah ho'tuun «y entonces ocurrió que en 1 Ajaw 13 Kumk'uh fue vista la atadura de piedra del primer ho 'tuun»; en la Estela 7 (E4-E5), el texto dice [i]-u-ti 3-TZIKIN 13-HULOL IL-B'AH, i u [h]ti 3 Tzikin 13 Hul O'hl ilb'aaj «y entonces ocurrió que en 3 Men 13 Kumk'uh (fue) la visión», o bien [i]-u-ti 3-TZIKIN 13-HUL-OL IL-[u]-B'AH, i u[h]ti 3 Tzikin 13 Hul O'hl il[aj] ub'aah «entonces ocurrió que en 3 Men 13 Kumk'uh fue vista su imagen ${ }^{28}$.

13 Ajaw 13 Kumk'uh (Estela 18), 9.18.10.7.5 12 Chikchan 13 Kumk'uh (Estela 2), 9.19.5.11.0 1 Ajaw 13 Kumk'uh (Estela 3), 9.19.10.12.0 1 Ajaw 8 K'umk'uh (Estela 4), 9.19.15.13.0 1 Ajaw 3 Kumk'uh (Estela 8), 10.0.0.14.15 3 Men 13 Kumk'uh (Estela 7), 10.0.5.16.0 8 Ajaw 13 Kumk'uh (Estela 6) y 10.0.10.17.5 13 Chikchan 13 Kumk'uh (Estela 5).

${ }^{25}$ Para un nuevo dibujo de estos bloques, vid. Lacadena 2005: Fig. 2.

${ }^{26}$ En yucateco colonial, way significa «celda, aposento, cámara, compartimiento» (Barrera 1980: 915). Houston (com. pers. a B. Just en septiembre de 2004, en Just 2006: Nota 157) y Boot (2005: 58-59) consideran asimismo que estos eventos se refieren a un escondite ceremonial.

${ }^{27}$ El sustantivo que nombra los propios recintos cuadrilobulados termina en /n/, como indica claramente el complemento final -na que suele llevar en ocasiones su logograma. Recientemente, Stuart ha sugerido para el conjunto completo T510-na-HA' una lectura pan-ha' «cavidad de agua» (mencionado por Carter y Houston, en Finamore y Houston 2010: 87), posiblemente relacionado con el verbo ch'orti' pajn «escarbar, cavar».

${ }^{28}$ Las diferencias en la lectura dependen en la ausencia o presencia de un signo u delante de B'AH, ambiguo en el dibujo de Graham de la Estela 7 (1967: 57). 
Boot $(2005: 56,59)$ y Just $(2006: 100,125)$ sugieren que lo que es visto son las propias dedicaciones de los monumentos, o la imagen de los gobernantes representados en ellos. En un caso análogo, en la Estela 9 de Seibal, donde se representa al gobernante wa-t'u-lu-k'a-te-le sujetando una barra ceremonial celeste de la que sobresalen la cabeza y la cola de una serpiente con los atributos de la Serpiente de Agua (Schele y Mathews 1998: 189-190; Boot 2005: 67; Just 2006: 247-248), el texto de la inscripción (B1-B2) dice: IL-u-B' AH-hi ti-K' AN-na WITZ' NAH-KAN-na, $i l[a j] ~ u b{ }^{\prime} a a h t i$ k'an Witz'Naah Kan «fue vista su imagen con la preciosa Witz' Naah Kan»-el nombre de la Serpiente de Agua -29 . Es muy posible que en Machaquilá el sentido preciso de estas expresiones con $i l$ «ver» se refieran no tanto al monumento o a la imagen de los gobernantes solos, sino que incidan en su aparición con la Serpiente de Agua. En ambos casos en las estelas 3 y 7 de Machaquilá, los gobernantes están personificando a la Serpiente de Agua, a quien posiblemente se ha conjurado como parte del ritual.

En resumen, la Plaza A fue uno de los espacios más importantes de actividad ritual de los gobernantes de Machaquilá, y de más continuidad en el tiempo. Sus características espaciales hablan de ritos de acceso restringido, en los que la élite de Machaquilá es la principal protagonista, como actante y asistente. El culto a las deidades del agua, Chaahk y Witz' Naah Kan, cuyas imágenes son conjuradas y personificadas por los gobernantes, así como el culto a los antepasados reales, posiblemente enterrados en las estructuras piramidales que flanquean la plaza en sus lados norte y este, incluyeron un complejo ceremonial centrado en el recinto cuadrilobulado, en el que los bailes y los ritos de fuego (asociados posiblemente a un ciclo recurrente de 3x365 días) involucraron una gran cantidad de objetos ceremoniales, ofrendas y posiblemente sacrificios humanos. Hacia mediados del siglo IX, la actividad ceremonial en la Plaza A cesó, tras la desaparición de la dinastía real.

\section{Referencias bibliográficas}

ArZÁPAlo MARín, Ramón

1995 Diccionario de Motul. Diccionario Maya-Español. Tomo I. México D. F.: Universidad Nacional Autónoma de México.

BArrera VÁsquez, Alfredo (ed.)

1980 Diccionario Maya Cordemex. Maya-Español, Español-Maya. Mérida: Ediciones Cordemex.

BARrientos, Tomás y Arthur Demarest

2007 «Cancuen: Puerta del mundo Maya Clásico», en XX Simposio de Investigaciones Arqueológicas en Guatemala, 2006, J.P. Laporte, B. Arroyo y H. Mejía, eds., pp.

\footnotetext{
${ }^{29}$ Stuart (2007), a quien se debe el desciframiento del logograma WITZ' «cascada»?, «salpicadura»?, sugiere leer el logograma NAH en este contexto como noh «grande» (como el mismo Stuart señala, entre los ch'ort'i' de Guatemala existe un ser sobrenatural comparable llamado noh chan «gran serpiente»), por lo que el nombre de la llamada Serpiente de Agua en el Clásico sería Witz’ Noh Kan «Gran Serpiente de la Cascada». NAH como naah «casa» podría hacer también sentido: el nombre se traduciría como «La Serpiente de la Casa de la Cascada» (la serpiente que habita en la Casa de la Cascada). En cualquier caso, es claramente un teónimo muy antiguo, fosilizado, que utiliza la forma kan «serpiente» (complementada normalmente como ka-KAN) en vez de chan.
} 
737-755. Guatemala: Museo Nacional de Arqueología y Etnología.

Barrientos, Tomás, Arthur Demarest, Silvia Alvarado, Horacio Martínez, Marc Wolf y Luis Fernando LuIN

2006 «Hidráulica, ecología, ideología y poder: Nueva evidencia y teorías en el sur de Petén», en XIX Simposio de Investigaciones Arqueológicas en Guatemala, 2005, J.P. Laporte, B. Arroyo y H. Mejía, eds., pp. 319-332. Guatemala: Museo Nacional de Arqueología y Etnología.

Boot, Erik

2002 «The Life and Times of B'alah Chan K'awil of Mutal (Dos Pilas), According to Dos Pilas Hieroglyphic Stairway 2». Documento electrónico, <www.mesoweb. com/features/boot/DPLHS2.pdf>, con acceso el 2/11/2010.

2005 Continuity and Change in Text and Image at Chichén Itzá, Yucatán, México. A Study of the Inscriptions, Iconography and Architecture at a Late Classic to Early Postclassic Maya Site. Leiden: CNWS Publications.

Chocón, Jorge E.

2003 «Machaquilá, un sitio abandonado por la Arqueología», en XVI Simposio de Investigaciones Arqueológicas en Guatemala, 2002, J.P. Laporte, B. Arroyo, H. Escobedo y H. Mejía, eds., pp.108-120. Guatemala: Museo Nacional de Arqueología y Etnología.

Chocón, Jorge E., M Josefa Iglesias, Alfonso LaCAdena y Jesús AdÁneZ

2007 «Excavaciones en Machaquilá, Petén: Temporada de campo 2005», en XX Simposio de Investigaciones Arqueológicas en Guatemala, 2006, J.P. Laporte, B. Arroyo y H. Mejía, eds., pp. 679-698. Guatemala: Museo Nacional de Arqueología y Etnología.

Chocón, Jorge E. y Juan Pedro LAPORTE

2002 «La ciudad de Machaquilá», en Reconocimientos y excavaciones arqueológicas en los municipios de Melchor de Mencos, Dolores, Poptun y San Luis, Petén, J. P. Laporte, ed., pp. 1-32. Reporte 16. Guatemala: Ministerio de Cultura y Deporte - Instituto de Antropología e Historia - Universidad de San Carlos.

Ciudad Ruiz, Andrés, Ma Josefa Iglesias, Jesús AdÁnez y Alfonso LaCAdEnA

2004 «Investigaciones arqueológicas en Machaquilá: la morada de \#-TI'-CHAHK-ki, príncipe de la tierra». Revista Española de antropología Americana 34: 29-62.

Ciudad Ruiz, Andrés, Alfonso Lacadena, Jesús AdÁnez y Ma Josefa Iglesias

2010 «Espacialidad y ritual en Machaquilá, Petén, Guatemala», en El ritual en el mundo maya: de lo privado a lo publico, A. Ciudad, Ma J. Iglesias y M. Sorroche, eds., pp. 129-151. Madrid: Sociedad Española de Estudios Mayas, Grupo de Investigación Andalucía-América, Centro Peninsular en Humanidades y Ciencias Sociales.

Ciudad Ruiz, Andrés, y Alfonso Lacadena García-Gallo

2006 «La fundación de Machaquilá, Petén, en el Clásico Tardío maya», en Nuevas ciudades, nuevas patrias: fundación, refundación y relocalización de las ciudades en Mesoamérica y el Mediterráneo antiguo, Mª J. Iglesias y R. Valencia, eds., pp. 149-180. Madrid: Sociedad Española de Estudios Mayas.

Demarest, Arthur., Tomás BARrientos y Federico FAHSEN

2006 «El apogeo y el Colapso del reinado de Cancuén: Resultados e interpretaciones del Proyecto Cancuen, 2004-2005», en XIX Simposio de Investigaciones Arqueológicas en Guatemala, 2005, J.P. Laporte, B. Arroyo y H. Mejía, eds., pp.826-837. Guatemala: Museo Nacional de Arqueología y Etnología. 
DemAREST, Arthur, y Federico FAHSEN

2003 «Nuevos datos e interpretaciones de los reinos occidentales del Clásico Tardío: hacia una visión sintética de la historia Pasión/Usumacinta», e n XVI Simposio de Investigaciones Arqueológicas en Guatemala, 2002, B. Arroyo, H. Escobedo, D. Guerra de González, Olga de Hazard, J. P. Laporte, F. Moscoso y Z. Rodríguez, eds., pp. 159-174. Guatemala: Ministerio de Cultura y Deportes, Instituto de Antropología e Historia, Asociación Tikal.

FAHSEN, Federico

1983 «Notas sobre la secuencia dinástica de Machaquilá». Mesoamérica 6: 417-433.

1984 «The Dynastic Sequence of Machaquila». American Antiquity 49 (1): 94-104.

2002 «Rescuing the Origins of Dos Pilas Dynasty: A Salvage of Hieroglyphic Stairway 2, Structure L5-49». Documento electrónico, <www.famsi.org/reports/01098/ index.html>, con acceso el 2/11/2010.

FAHSEn, Federico, Jeannette CASTEllanos, Jorge Mario OrTiz y Fernando Luin

2003 «La Escalinata 2 de Dos Pilas, Petén: los nuevos escalones», en XVI Simposio de Investigaciones Arqueológicas en Guatemala, 2002, B. Arroyo, H. Escobedo, D. Guerra de González, Olga de Hazard, J. P. Laporte, F. Moscoso y Z. Rodríguez, eds., pp. 687-700. Guatemala: Ministerio de Cultura y Deportes, Instituto de Antropología e Historia, Asociación Tikal.

FAHSEN, Federico, y Arthur Demarest

2001 «El papel del reino de Cancuén en la historia de las Tierras Bajas Mayas. Nuevos datos epigráficos», en XIV Simposio de Investigaciones Arqueológicas en Guatemala, 2000, J.P. Laporte, A.C. Suasnávar y B. Arroyo, eds., pp. 999-1015. Guatemala: Museo Nacional de Arqueología y Etnología.

FAHSEn, Federico, Arthur Demarest y Luis F. Luin

2003 «Sesenta años de historia en la Escalinata Jeroglífica de Cancuén», en XVI Simposio de Investigaciones Arqueológicas en Guatemala, 2002, J. P. Laporte, B. Arroyo, H. L. Escobedo y H. E. Mejía, eds., pp. 711-722. Guatemala: Museo Nacional de Arqueología y Etnología.

FAHSEN, Federico, y Sarah JACKSON

2002 «Nuevos datos e interpretaciones sobre la dinastía de Cancuén en el Periodo Clásico», en XV Simposio de Investigaciones Arqueológicas en Guatemala, 2001, J. P. Laporte, H. Escobedo y B. Arroyo, eds., pp. 889-908. Guatemala: Museo Nacional de Arqueología y Etnología.

FASH, William, Alexandre ToKOVININE, y Barbara FASH

2009 «The House of the New Fire at Teotihuacan and its Legacy in Mesoamerica», en The Art of Urbanism: How the Mesoamerican Kingdoms Represented Themselves in Architecture and Imagery, W. Fash y L. López Luján, eds., pp. 201-229. Washington: Dumbarton Oaks Research Library and Collection.

FinAmore, Daniel, y Stephen Houston (eds.)

2010 Fiery Pool. The Maya and the Mythic Sea. New Haven y Londres: Peabody Essex Museum, Yale University Press.

GARCía BARrios, Ana

2009 Chaahk, el dios de la lluvia, en el Periodo Clásico Maya: aspectos políticos y religiosos. Madrid: E-Prints Complutense. Documento electrónico, <http://eprints. 
ucm.es/view/people/Garc=EDa_Barrios,_Ana.html>, con acceso el 2/11/2010.

GRAHAM, Ian

1967 Archaeological Explorations in El Peten, Guatemala. Middle American Research Institute, Publ. 33. New Orleans: Tulane University.

GRUBE, Nikolai

1989 «Archaeological investigations in the Southern Peten (Machaquila, San Miguel)». Mexicon 11 (3): 44-45.

GUENTER, Stanley

2002 Under a Falling Star: The Hiatus at Tikal. Tesis de maestría. La Trobe University.

2003 «The Inscriptions of Dos Pilas Associated with B'ajlaj Chan K'awiil». Documento electrónico, <www.mesoweb.com/features/guenter/Dos Pilas.pdf>, con acceso el $2 / 11 / 2010$.

Houston, Stephen

1992 «La historia de Dos Pilas y de sus gobernantes». Apuntes Arqueológicos 2 (2): 1546.

1993 Hieroglyphs and History at Dos Pilas: Dynastic Politics of the Classic Maya. Austin: University of Texas Press.

Houston, Stephen, y Takeshi InOMATA

2009 The Classic Maya. Cambridge: Cambridge University Press.

Houston, Stephen, y Simon MarTin

2010 «Let the Glyphs Be Few: Abbreviations in Maya Writing». Documento electrónico, $<$ http://decipherment.wordpress.com/2011/01/16/let-thy-glyphs-be-fewabbreviations-in-maya-writing/>, con acceso el 21/1/2011.

Houston, Stephen, and Peter Mathews

1985 The Dynastic Sequence of Dos Pilas, Guatemala. Pre-Columbian Art Research Institute, Monograph 1. San Francisco: Pre-Columbian Art Research Institute.

Houston, Stephen D., y David StuART

2001 «Peopling the Classic Maya Court», en Royal Courts of the Ancient Maya, Volume One: Theory, Comparison, and Synthesis, T. Inomata y S. Houston, eds., pp. 5483, Boulder: Westview Press.

Houston, Stephen, Zachary Nelson, Gene Ware, Cassandra Mesick, Karl Taube, Ray MATHENY y Deanne MATHENY

2005 «The Pool of the Rain God. An early Stuccoed Altar at Aguacatal, Campeche, Mexico». Mesoamerican Voices 2.

Iglesias Ponce de León, Ma Josefa, y Alfonso LaCAdena García-Gallo

2003 «Nuevos hallazgos glíficos en la Estructura 4 de Machaquilá, Petén, Guatemala». Mayab 16: 65-71.

INOMATA, Takeshi

1997 «The Last Day of a Fortified Classic Maya Center: Archaeological Investigations at Aguateca, Guatemala». American Antiquity 8 (2): 337-351.

InOmata, Takeshi, Erik Ponciano, Oscar SAntos, Oswaldo Chinchilla, Otto Román y

Verónica BREUIL-MARTÍNEZ

2004 «An Unfinished Temple at the Classic Maya Center of Aguateca, Guatemala». Antiquity 78 (302): 798-811. 
InOMATA, Takeshi, y Daniela TRIADAN (eds.)

2010 Burned Palaces and Elite Residences of Aguateca: Excavations and Ceramics. Monographs of the Aguateca Archaeological Project First Phase, Vol. 1. Salt Lake City: University of Utah Press.

Just, Bryan

2006 The Visual Discourse of Ninth-Century Stelae at Machaquila and Seibal. Tesis doctoral inédita. University of Tulane.

KistLer, Ashley

2004 «The Search for Five-Flower Mountain: Re-Evaluating the Cancuen Panel». Documento electrónico, <www.mesoweb.com/features/kistler/Cancuen.pdf $>$, con acceso el 2/11/2010.

LACADENa García-Gallo, Alfonso

2003 «El corpus glífico de Ek’ Balam, Yucatán, México / The Glyphic Corpus of Ek' Balam, Yucatan, Mexico». FAMSI Reports: Documento electrónico, <http://www.famsi.org/reports/0157es/index.htlm>, con acceso el 2/11/2010.

2005 «Excavaciones en Machaquila, Poptun: Estudio epigráfico realizado durante la temporada 2004», en Reporte 19, Atlas Arqueológico de Guatemala, J. P. Laporte, ed., pp.235-247. Guatemala: Instituto de Antropología e Historia, Guatemala.

2006 «Excavaciones en Machaquilá, Temporada 2005: El recinto cuadrilobulado de la Plaza A», en Reporte 20, Atlas Arqueológico de Guatemala: exploraciones arqueológicas en el sureste y centro-oeste de Petén, J. P. Laporte y H. Mejía, eds., pp. 74123. Guatemala: Dirección general del Patrimonio Cultural y Natural, Ministerio de Cultura y Deportes.

LaCAdena García-Gallo, Alfonso, y Andrés Ciudad Ruiz

1998 «Reflexiones sobre la estructura política maya clásica», en Anatomía de una civilización: aproximaciones disciplinares a la cultura maya, A. Ciudad, Y. Fernández, J.M. García, M.J. Iglesias, A. Lacadena, y L. Sanz, eds., pp. 31-64. Madrid: Sociedad Española de Estudios Mayas.

Lacadena García-Gallo, Alfonso, y Ma Josefa Iglesias Ponce De León

2005 «Una relación epigráfica relacionada con la Estructura 4 de Machaquilá», en XVIII Simposio de Investigaciones Arqueológicas en Guatemala, 2004, J. P. Laporte, B. Arroyo y H. Mejía, eds., pp. 677-690. Guatemala: Ministerio de Cultura y Deportes, Instituto de Antropología e Historia, Asociación Tikal, Foundation for the Advancement of Mesoamerican Studies, Inc.

Love, Michael, Julia Guernsey, Sheryl Carcuz y Molly Morgan

2006 «El Monumento 3 de La Blanca: Una Nueva Escultura del Preclásico Medio», en XIX Simposio de Investigaciones Arqueológicas en Guatemala, 2005, J. P. Laporte, B. Arroyo y H. Mejía, eds. Guatemala: Museo Nacional de Arqueología y Etnología.

Martin, Simon, y Nikolai Grube

1994 «Evidence for Macro-political Organization Amongst Classic Maya Lowland States». Manuscrito.

1995 «Maya Superstates». Archaeology 48 (6): 41-46.

2000 Chronicle of the Maya Kings and Queens: Deciphering the Dynasties of the Ancient Maya. Londres: Thames and Hudson. 
Mathews, Peter

1991 «Classic Maya Emblem Glyphs», en Classic Maya Political History. Hieroglyphic and Archaeological Evidence, T. P. Culbert, ed., pp. 19-29. Cambridge: Cambridge University Press.

Mathews, Peter, y Gordon WiLley

1991 «Prehistoric Polities of the Pasion Region: Hieroglyphic Texts and their Archaeological Settings», en Classic Maya Political History: Hieroglyphic and Archaeological Evidence, T. P. Culbert, ed., pp. 30-71. Cambridge: Cambridge University Press.

MAYER, Karl H.

1987 Maya Monumnets: Sculptures on Unknown Provenance (IV). Berlín: Verlag von Flemming.

NiELSEN, Jesper

2003 Art of the Empire: Teotihuacan Iconography and Style in Early Classic Maya Society (A.D. 380-500). Tesis doctoral inédita. University of Copenhagen.

ProskouriakofF, Tatiana

1950 A Study of Classic Maya Sculpture. Publication 593. Washington: Carnegie Institution of Washington.

REENTS-BudET, Dorie

1991 «The «Holmul Dancer» Theme in Maya Art», en VI Palenque Round Table, V. M. Fields, ed., pp. 217-222. Norman: University of Oklahoma Press.

1994 Painting the Maya Universe: Royal Ceramics of the Classic Period. Durham y Londres: Duke University Press.

RIESE, Berthold

1984 «Dynastiegeschichtliche und kalendarische Beobachtungen an den Maya-Inschriften von Machaquilá, Petén, Guatemala». Tribus 33: 149-154.

1988a «Neues zur Dynastie und Chronologie von Machaquila». Mexicon 19 (5): 96-98.

1988 bWeiteres zur Dynastie und Chronologie von Machaquilá». Tribus 37: 175-177.

SATURno, William, David StuART y Karl TAube

2005 «La identificación de las figuras del Muro Oeste de Pinturas Sub-1, San Bartolo, Petén», en XVIII Simposio de Investigaciones Arqueológicas en Guatemala, 2004, J. P. Laporte, B. Arroyo y H. Mejía, eds., pp.626-635. Guatemala: Museo Nacional de Arqueología y Etnología.

SCHELE, Linda, y Nikolai GRUBE

1994 Notebook for the XVIII Hieroglyphic Workshop at Texas. Austin: The Center for Mexican Studies and Institute of the Latin American Studies, University of Texas.

1995 Notebook for the XIX Hieroglyphic Workshop at Texas. Austin: The Center for Mexican Studies and Institute of the Latin American Studies, University of Texas.

Schele, Linda, and Peter Mathews

1998 The Code of Kings: The Language of Seven Sacred Maya Temples and Tombs. Nueva York: Scribner.

STUART, David

2000 "The Arrival of Strangers': Teotihuacan and Tollan in Classic Maya History», en Mesoamerica's Classic Heritage, D. Carrasco, L. Jones y S. Sessions, eds., pp. 465-513. Niwot: University Press of Colorado. 
2007 «Reading the Water Serpent as WITZ'. Maya decipherment. A Weblog on the Ancient Maya Script». Documento electrónico, $<$ http://decipherment.wordpress.com/ ?s=witz $\% 27+$ noh $>$, con acceso el 2/11/2010.

STUART, David, y Stephen Houston

1994 Classic Maya Place Names. Studies in Pre-Columbian Art and Archaeology 33. Washington: Dumbarton Oaks.

TOMASIC, John, y Federico FAHSEN

2004 «Exploraciones y excavaciones preliminares en Tres Islas, Petén», en XVII Simposio de Investigaciones Arqueológicas en Guatemala, 2003, J. P. Laporte, B. Arroyo, H. Escobedo y H. Mejía, eds., pp. 819-832. Guatemala: Ministerio de Cultura y Deportes, Instituto de Antropología e Historia, Asociación Tikal.

TOMAsic, John, Claudia M. QuintANILla y Edy BARrios

2005 «Excavaciones en el sitio Arqueológico Tres Islas, Río Pasión, Petén», en XVIII Simposio de Investigaciones Arqueológicas en Guatemala, 2004, J. Pedro Laporte, Bárbara Arroyo y Héctor E. Mejía, eds., pp. 403-412. Guatemala: Ministerio de Cultura y Deportes, Instituto de Antropología e Historia, Asociación Tikal, Foundation for the Advancement of Mesoamerican Studies, Inc.

ZENDER, Marc

2004 «New Discoveries at Cancuen». Documento electrónico, <www.mesoweb.com/ reports/cancuen_new.html>, con acceso el 2/11/2010.

2005 «Teasing the Turtle from its Shell: AHK and MAHK in Maya Writing». The PARI Journal 6 (3):1-14.

ZENDER, Marc, y Joel SKIDMORE

2004 «New Ballcourt Marker from Cancuen». Documento electrónico, <www. mesoweb.com/reports/cancuen_altar.html>, con acceso el 2/11/2010.

\section{Apéndice I: Relación de textos jeroglíficos de Machaquilá}

ALTAR A

Ubicación original: Plaza A, al oeste del eje del recinto cuadrilobulado.

Ubicación actual: Desconocida. Restos mutilados con sierra mecánica esparcidos en el sitio.

Documentación: Foto en Graham 1967: Fig. 73.

ALTAR B

Ubicación original: Plaza A, al pie de la Estructura 20.

Ubicación actual: Desconocida..

Documentación: Dibujo en Graham 1967: Fig. 76.

ESTELA 1

Ubicación original: Plaza A, al pie de la Estructura 20.

Ubicación actual: Desconocida. Restos de la parte posterior sin relieve en el sitio.

Documentación: Referencia en Graham 1967: 59, 60, quien la registra como plana (pero vid. Chocón y Laporte 2002: 12).

ESTELA 2

Ubicación original: Plaza A, al pie de la Estructura 20.

Ubicación actual: El frente se encuentra en el Museo de la Aurora de Guatemala. Se desconoce el paradero de la parte trasera, saqueada con sierra mecánica. 
Documentación: Dibujos en Graham 1967: Fig. 44 (frente), Fig. 47 (lados); foto de la espalda con glifos en Graham 1967: Fig. 45 (espalda). Fotos de los restos mutilados en Chocón y Laporte 2002: 13.

\section{ESTELA 3}

Ubicación original: Plaza A, al pie de la Estructura 20.

Ubicación actual: Museo de La Aurora, Ciudad de Guatemala.

Documentación: Dibujo en Graham 1967: Fig. 49.

\section{ESTELA 4}

Ubicación original: Plaza A, al pie de la Estructura 20.

Ubicación actual: Museo de La Aurora, Ciudad de Guatemala.

Documentación: Dibujo en Graham 1967: Fig. 51.

\section{ESTELA 5}

Ubicación original: Plaza A, al pie de la Estructura 19.

Ubicación actual: Bodega del Museo de La Aurora, Ciudad de Guatemala.

Documentación: Dibujo en Graham 1967: Fig. 53, y boceto revisado de los bloques A3-A4 en Lacadena 2005: Fig. 2.

\section{ESTELA 6}

Ubicación original: Plaza A, al pie de la Estructura 19.

Ubicación actual: Mutilada y saqueada. Fragmentos rescatados en la Bodega del Museo de La Aurora, Ciudad de Guatemala.

Documentación: Dibujos: Graham 1967: Fig. 55.

\section{ESTELA 7}

Ubicación original: Plaza A, al pie de la Estructura 19.

Ubicación actual: Museo de La Aurora, Ciudad de Guatemala.

Documentación: Dibujo en Graham 1967: Fig. 57.

\section{ESTELA 8}

Ubicación original: Plaza A, al pie de la Estructura 20.

Ubicación actual: Restos mutilados en el sitio. El frente se recuperó y se encuentra en la Asociación Tikal, Ciudad de Guatemala (Chocón y Laporte 2002: 16).

Documentación: Dibujo en Graham 1967: Fig. 59.

\section{ESTELA 9}

Ubicación original: Plaza A, al pie de la Estructura 19.

Ubicación actual: La misma.

Documentación: Graham 1967: 77. Plana

\section{ESTELA 10}

Ubicación original: Plaza A, al pie de la Estructura 17.

Ubicación actual: Mutilada, en el sitio sólo se encuentran restos sin relieve.

Documentación: Dibujo en Graham 1967: Fig. 61.

\section{ESTELA 11}

Ubicación original: Plaza A, al pie de la Estructura 17.

Ubicación actual: Desconocida.

Documentación: Dibujo en Graham 1967: Fig. 63.

\section{ESTELA 12}

Ubicación original: Plaza A, al pie de la Estructura 17.

Ubicación actual: Mutilada, en el sitio sólo quedan restos de la parte inferior.

Documentación: Dibujo en Graham 1967: Fig. 65.

ESTELA 13

Ubicación original: Plaza A, al pie de la Estructura 17. 
Ubicación actual: Bodega del Museo de La Aurora, Ciudad de Guatemala.

Documentación: Dibujo en Graham 1967: Fig. 67.

ESTELA 14

Ubicación original: Plaza A, al pie de la Estructura 16.

Ubicación actual: La misma.

Documentación: Graham 1967: 88-89. Plana?

ESTELA 15

Ubicación original: Plaza A, al pie de la Estructura 16.

Ubicación Actual: La misma.

Documentación: Graham 1967: 89. Plana.

ESTELA 16

Ubicación original: Plaza A, al pie de la Estructura 16.

Ubicación actual: La misma.

Documentación: Graham 1967: 89. Plana?

\section{ESTELA 17}

Ubicación original: Plaza C, en el eje este-oeste, al sur de la Estructura 26.

Ubicación actual: La misma.

Documentación: Graham 1967: 89-92; fotografía en Graham 1967: Fig. 70, y en Chocón y Laporte 2002: 20.

ESTELA 18

Ubicación original: Plaza C, en el eje este-oeste, al pie de la Estructura 36 (vid. Just 2006: Cap. 2, Nota 108).

Ubicación actual: Colección privada en Europa.

Documentación: Dibujos en Fahsen 1984: Fig. 5, y Riese 1988a: 97. Fotografía en Mayer 1987: Pl. 40 y 41.

ESTELA 19

Ubicación original: Plaza C, en el eje este-oeste, al pie de la Estructura 36 (vid. Just 2006: Cap. 2, Nota 108 y 123).

Ubicación actual: aproximadamente la misma, aunque ha sido erigida.

Documentación: Fotos y boceto de campo de A. Lacadena.

ESTELA 20

Ubicación original: Plaza D, a la derecha de la escalinata de la Estructura 34.

Ubicación actual: Mutilada, sólo queda en el sitio la parte posterior sin relieve.

Documentación: Chocón y Laporte 2002: 23.

ESTELA 21

Ubicación original: Plaza D, a la izquierda de la escalinata de la Estructura 34.

Ubicación actual: Mutilada, sólo queda en el sitio la parte posterior sin relieve.

Documentación: Chocón y Laporte 2002: 23.

ESTELA 22

Ubicación original: Extremo sur de la Plaza D.

Ubicación actual: Mutilada, queda en el sitio la parte posterior con restos de relieve en los laterales.

Documentación: Chocón y Laporte 2002: 24.

FRAGMENTOS GLÍFICOS DE LAS ESTRUCTURAS 4 Y 10 Y ESPALDA DE LA 7-8

Ubicación original: Plaza F, estructuras 4, 10 y 7-8.

Ubicación actual: de algunos fragmentos se desconoce; otros presumiblemente saqueados; otros en la Bodega del Atlas Arqueológico de Guatemala (Dolores, Petén) y Museo de Dolores.

Documentación: Fotos y dibujos de 21 fragmentos (A-U) en Graham 1967: Fig. 39; dibujo de un fragmento en Grube 1988: Fig. 2; fotos y/o dibujos de 19 nuevos fragmentos en Iglesias y Lacadena 2003:

Figs. 4-8, Lacadena 2005: Figs. 4b, 5, 6, 9 y Lacadena e Iglesias 2005: Fig. 2a-p. 
Nota adicional: Guido Krempel (com. pers., diciembre de 2010 y enero de 2011), ha informado del hallazgo en agosto de 2009 de tres nuevos fragmentos integrando un mismo bloque con tres bloques jeroglíficos, posiblemente procedentes de la Plaza F.

\section{FRAGMENTOS GLÍFICOS CERÁMICOS}

Ubicación original: Plaza A, recinto cuadrilobulado.

Ubicación actual: Bodega del Atlas Arqueológico de Guatemala, Dolores.

Documentación: Fotos y dibujos en Lacadena 2006: Fig 18.

\section{Apéndice II: Secuencia dinástica de Machaquilá}

\begin{tabular}{lll}
\hline Nombre & Textos asociados* & Fechas asociadas \\
\hline [18 reyes tempranos] & TRS, E2 & ca. ss. II-V d.C. \\
Yax Tzu'? Chaahk & TRS, E1 y 2 & 475 d.C. \\
Tajal Mo' ?** & DPL, EJ2, EJ4, P7 & 664 d.C. \\
Siyaj K'in Chaahk I & MQL, E11, 13?, 14?, 15?, 16? & 711 d.C.? \\
«Etz'nab'» Chaahk & MQL, E10, 11, 12, 13?, 17? & $711 ?, 731 ?, 741,761$ d.C. \\
Chak ... B'ahlam & MQL, E18?, 19? & 775,776 d.C. \\
Tajal Chan Ahk de Cancuén & CNC, EJ1, P3 y 160g, MJP2 & $786 ?, 790 ?, 795,799$ d.C. \\
K'an Maax de Cancuén & CNC, MJP3 & $c a .895$ d.C. \\
Ochk'in Kalo'mte' & MQL, E1? y 2 & $770,799,800,801$ d.C. \\
Siyaj K'in Chaahk II & MQL, E3 y 4 & $815,816,820,821$ d.C. \\
uchan ...b'ul K'ahk'? & MQL, E6 & $c a .821-824 ?$ \\
Jun Tzak Tok' & MQL E 5, 6, 7, 8, 9? & $824,825,830,831,835,836,840$ d.C. \\
«Escorpión» Ti' Chaahk & MQL, Estr. 4, Frags. (BJ1?) & $>840$ d.C.? \\
\hline Abreviaturas
\end{tabular}

*Abreviaturas empleadas: $\mathrm{TRS}=$ Tres Islas; $\mathrm{DPL}=$ Dos Pilas; $\mathrm{MQL}=$ Machaquilá $\mathrm{CNC}=$ Cancuén; $\mathrm{E}=$ Estela; EJ= Escalera Jeroglífica; $\mathrm{P}=$ Panel; $\mathrm{MJP}=$ Marcador de Juego de Pelota; BJ= Banca Jeroglífica.

${ }^{* *}$ Tajal Mo' es aparentemente un título genérico para cautivos. No es seguro que fuera gobernante de Machaquilá. 